\title{
Tunneling of a few strongly repulsive hard-sphere bosons in an optical lattice with tight external harmonic confinement: A quantum Monte Carlo investigation in continuous space
}

\author{
Asaad R. Sakhel, ${ }^{1}$ Jonathan L. Dubois, ${ }^{2}$ and Roger R. Sakhel ${ }^{3}$ \\ ${ }^{1}$ Al-Balqa Applied University, Faculty of Engineering Technology, \\ Basic Sciences Department, Amman 11134, JORDAN \\ ${ }^{2}$ Lawrence Livermore National Lab, 7000 East Ave, L-415, Livermore CA 94550, USA \\ ${ }^{3}$ Department of Basic Sciences, Al-Isra Private University, Amman 11622, JORDAN
}

(Dated: September 14, 2021)

\begin{abstract}
The effect of strongly repulsive interactions on the tunneling amplitude of hard-sphere (HS) bosons confined in a simple cubic (sc) optical lattice plus tight external harmonic confinement in continuous space is investigated. The quantum variational Monte Carlo (VMC) and the variational path integral Monte Carlo (VPI) techniques are used at zero temperature. The effects of the lattice spacing $\pi / k$ on the tunneling amplitude is also considered. The occupancies of the lattice sites as a function of the repulsion between the bosons are further revealed. Our chief result is, that for a small number of bosons $(\mathrm{N}=8)$ the overlap of the wave functions in neighboring wells does not change with an increase of the repulsive interactions and changes only minimally for a larger number of particles $(N=40)$. The tunneling amplitude rises with a reduction in the lattice spacing. In addition, the occupancy of the center of the trap decreases in favor of a rise in the occupancy of the lattice sites at the edges of the trap with increasing HS repulsion. Further, it was found that the energy per particle at certain optical depths is insensitive to the number of particles and variations in the HS diameter of the bosons. In order to support our results, we compare the VMC results with corresponding VPI results.
\end{abstract}

\section{INTRODUCTION}

The tunneling of bosons in optical lattices has drawn considerable interest in the last few years [1, 2, 3, 4, 15, 6, 7, 8, 9, 10, 11 due to the fact the investigation of the tunneling amplitude in optical lattices provides insight into the physics of lattice bosons. This is due to its connection to superfluidity [12] and its analogy to Josephson tunneling in quantum devices [13. Particularly the role of interparticle interactions in determining the tunneling amplitude has only been given few investigations.

In simple elementary terms, the quantum tunneling of a particle through a potential barrier, such as in an optical lattice, can occur when its total energy $E$ is less than the height of the barrier $V_{0}$. The particle is described by a wave packet which can penetrate the barrier with a finite probability. As a result, an overlap between two wave functions at both sides of the barrier provides a measure for the tunneling amplitude of the particle. Potential barriers of this sort have been realized in quantum devices [13] that make use of Josephson tunneling [8] and in the recently achieved optical trapping of bosons 14. When there is more than a single atom in the potential well, the mechanism of quantum tunneling is very much determined by the strength of the interatomic interactions. In the strongly interacting regime, correlated hopping occurs [5, 15] where atoms tunnel in pairs and competes with single-particle tunneling.

Further, one of the most important properties of single-particle tunneling in optical lattices is that it is a signature of a superfluid (SF) state [12, and if absent, of a Mott-insulator (MI) state [4, 7, 16. Pair superfluidity has also been recently discussed [15]. In an MI state, single-particle tunneling and phase coherence are absent. Consequently, the particles are unable to superflow, but still able to hopp from one well to the other. This is via the correlated hopping mechanism signalled by an overlap of the wave functions in neighboring wells in the strongly interacting regime. In fact it was Fölling et al. [5] who showed experimentally that strong interactions suppress single-particle tunneling such that second-order correlated tunneling is then the dominant dynamical effect. An SF state is quite the opposite, where considerable single-particle tunneling and phase coherence are observed.

In this paper, we chiefly investigate the effects of strongly repulsive interactions on the correlated tunneling amplitude of bosons in an inhomogeneous system confined by a tight combined harmonic optical lattice. Here, the external harmonic trap introduces an inhomogeneiety in the atomic density distributions. The tunneling amplitude is measured by the overlap integral of two wave functions in neighboring wells obtained from the integrated optical densities. A key point in our investigation is to also explore the possibility of a SF to MI transition in the presence of this inhomogeneiety since it has been shown that external confinement added to the optical lattice suppresses the MI state [17, 18. We partly check this case for few-boson systems. We use quantum variational Monte Carlo (VMC) and variational path integral (VPI) Monte Carlo techniques in continuous space at zero Kelvin. To the best of our knowledge, previous work did not consider the effects of inhomogeneiety on the tunneling amplitude of strongly repulsive lattice bosons, particularly by using Monte Carlo techniques in continuous space. 
To this end, the tunneling amplitude of bosons in optical lattices has been investigated chiefly as a function of the optical depth (i.e., barrier height $V_{0}$ ) [5, 12, 19, and number of particles 7 . An investigation most relevant to our work is that of Shams and Glyde [12. They evaluated the BEC density and superfluid fraction of HS bosons confined in an external periodic potential using PIMC in order to shed further light on the connection between BEC and superfluidity. In part, they investigated the hopping parameter as a function of $V_{0}$, and showed that if $V_{0}$ is increased sufficiently, the condensate is localized into islands inside the potential wells suppressing superflow substantially. Further, they found that their external potential suppresses the superfluid fraction at all temperatures.

In our investigation a key point is that, in contrast to Shams and Glyde, $V_{0}$ is kept fixed while the repulsive interactions between the bosons are varied. Further, the effects of these interactions on atom correlations, optical density, occupancy of lattice sites, onsite interaction energies, and the total energies are explored. In addition, the effects due to lattice spacing and number of particles are further revealed.

As outlined in the method section, the bosons are represented by hard spheres (HS) of diameter $a_{c}$ whose repulsive interactions can be modified by changing $a_{c}$, thereby mimicking the Feshbach resonance technique 20]. Our chief result is that, for a small number of bosons, the overlap of wave functions in neighboring potential wells does not change with increasing HS repulsion and changes only minimally for $N=40$. The localized wave functions in the potential wells do not broaden with an increase in $a_{c}$, in contrast to the case of HS bosons in pure harmonic traps [21, 22]. In the latter, the width of the spacial many-body wave function extends to several trap lengths, whereas in our case, the wavefunction in each well extends only slightly beyond two trap lengths even at large repulsion. We thus have evidence to suggest, that the optical lattice barriers prevent the wave functions in each well from expanding to a comparable extent as in pure harmonic traps. We further found that the energy per particle is relatively insensitive to the number of particles $N$ and variations in $a_{c}$ as compared to HS bosons in pure harmonic traps [21, 22], and that the tunneling amplitude increases with the reduction of the lattice spacing. In order to provide further support to our findings, we compare our VMC results with the corresponding VPI results.

Previous theoretical work on optical lattices is abundant, particularly the SF to MI transition [7, 9, 16, 17, 23, $24,25,26$ and coherent matter waves [16, 27, 28, 29, 30 have been investigated extensively. Other investigations included supersolidity [31, multicomponent systems [32, 33], vortices [34, solitons in a radially periodic lattice [35], and p-h excitations in MIs [16].

Various techniques and methods have been used to investigate bosons, fermions, or mixtures of them in optical lattices: the Bose-Hubbard model [7, 17, 25, 36] as well as the Fermi-Hubbard model [37, 38] in conjunction with Monte Carlo techniques [17, 25, 26, 37, 39, and particularly the Worm Algorithm [38, 40, 41] have been applied extensively. Other important techniques such as the Gross-Pitaevskii equation (GPE) [3, 35, variational approaches [7, 23, 35, density matrix renormalization group [30, and path integral approaches [42] have also been used. Most of the above methods use a discrete space approach, whereas we evaluate the properties in continuous space.

\section{METHOD}

We thus consider $N$ bosons on a combined harmonic optical cubic lattice (CHOCL). It consists of a $3 \mathrm{D}^{a l}$ simple cubic (SC) lattice of $N_{L}=3 \times 3 \times 3$ sites embedded in a tight external harmonic trap of frequency $\omega_{h o}$ and trap length $a_{h o}=\sqrt{\hbar / m \omega_{h o}}$, where $m$ is the mass of the bosons, and $\hbar$ is Planck's constant. The lattice spacing is given by $d=\pi / k$, where $k$ is the wave vector of the laser light. The bosons are modelled by hard spheres (HS) of diameter $a_{c}$ and their interactions $V_{i n t}(r)$ are represented by a hard-core potential of diameter $a_{c}$ given by

$$
V_{\text {int }}(r)=\left\{\begin{array}{rll}
\infty & : & r \leq a_{c} \\
0 & : & r>a_{c}
\end{array},\right.
$$

where $r$ is the distance between a pair of bosons. In the low-energy limit, the scattering between the bosons is purely s-wave with scattering length $a_{s}$. In this limit, $a_{s}$ equals $a_{c}$ 21, 22, and the repulsive interactions between the bosons are modified by changing $a_{c}$. Within this framework then, the tunneling amplitude $J$ and the rest of the properties are measured as functions of $a_{c}$.

To set the stage, then, we first define the Hamiltonian, and the trial wave function. Then the tunneling amplitude is measured by the overlap integral $I_{\text {overlap }}$ of two wave functions in neighboring wells centered at positions $\mathbf{R}_{n}$ and $\mathbf{R}_{n+1}$ measured from the center of the trap, where $n$ is an arbitrary integer. Here $n \equiv(p q r)$ are site indices with locations $\mathbf{R}_{n}=(p \hat{\mathbf{i}}+q \hat{\mathbf{j}}+r \hat{\mathbf{k}})$ in units of the lattice spacing $d$. By evaluating the overlap integral, the purpose is just to obtain a qualitative measure for the tunneling amplitude. Next the average onsite interaction energy $\left\langle U_{n}\right\rangle$ and the average occupation number per lattice site $\left\langle N_{p q r}\right\rangle$ at position $\mathbf{R}_{n} \equiv(p q r)$ are further defined. These are evaluated using VMC and VPI. We do not explain the VMC and VPI methods here as they can be found in the abundant literature [21, 43, 44, 45].

\section{A. Hamiltonian}

The Hamiltonian for our systems is given by 
$H=-\frac{\hbar^{2}}{2 m} \sum_{i=1}^{N} \nabla_{i}^{2}+\sum_{i=1}^{N}\left[V_{h o}\left(\mathbf{r}_{i}\right)+V_{o p t}\left(\mathbf{r}_{i}\right)\right]+\sum_{i<j} V_{i n t}\left(r_{i j}\right)$,

where $V_{h o}\left(\mathbf{r}_{i}\right)=\frac{1}{2} m \omega_{h o}^{2} r_{i}^{2}$ is an external harmonic trapping potential with $\mathbf{r}_{i} \equiv\left(x_{i}, y_{i}, z_{i}\right)$ representing a single particle position, $m$ the mass of the bosons, $V_{i n t}\left(r_{i j}\right)$ with $r_{i j}=\left|\mathbf{r}_{i}-\mathbf{r}_{j}\right|$ the pair-interaction potential Eq.(1) above, and

$$
V_{\text {opt }}\left(\mathbf{r}_{i}\right)=V_{0}\left[\sin ^{2}\left(k x_{i}\right)+\sin ^{2}\left(k y_{i}\right)+\sin ^{2}\left(k z_{i}\right)\right],
$$

is the optical lattice potential [7] with $V_{0}$ the optical depth. Essentially, $V_{\text {opt }}\left(\mathbf{r}_{i}=\mathbf{R}_{n}\right)=0$ at the lattice-site positions $\mathbf{R}_{n}$ which are the locations of the potential-well minima of Eq. (3). That is, $V_{\text {opt }}\left(\mathbf{R}_{n}\right)=V_{0}\left[\sin ^{2}(p \pi)+\right.$ $\left.\sin ^{2}(q \pi)+\sin ^{2}(r \pi)\right]=0$ where $\mathbf{k} \cdot \mathbf{R}_{n}=$ (integer) $\times \pi$. Thus the lattice-site positions are implied in $V_{o p t}\left(\mathbf{r}_{i}\right)$ by its very construction. Experimentally, the optical lattice potential is obtained from a superposition of three pairs of mutually perpendicular, counterpropagating laser beams of intensity proportional to $V_{0}$. For a $\mathrm{SC}$ lattice, $V_{0}$ must be the same for each direction. In this paper, we write energy and length in units of the trap, $\hbar \omega_{h o}$ and $a_{h o}=\sqrt{\hbar / m \omega_{h o}}$, respectively.

\section{B. Trial wave function}

The many-body trial wave function is given by

$\Psi(\{\mathbf{r}\},\{\mathbf{R}\})=\prod_{i=1}^{N} \exp \left(-\alpha r_{i}^{2}\right) \psi\left(\mathbf{r}_{i},\{\mathbf{R}\}\right) \prod_{i<j} f\left(\left|\mathbf{r}_{i}-\mathbf{r}_{j}\right|\right)$,

with $\psi\left(\mathbf{r}_{i},\{\mathbf{R}\}\right)$ a Wannier-like function defined as

$$
\psi\left(\mathbf{r}_{i},\{\mathbf{R}\}\right)=\sum_{n=0}^{N_{L}} \phi\left(\mathbf{r}_{i}, \mathbf{R}_{n}\right),
$$

where $\{\mathbf{r}\} \equiv\left\{\mathbf{r}_{1}, \mathbf{r}_{2}, \cdots, \mathbf{r}_{N}\right\}$ is a set of spatial vectors describing the positions of the bosons from the center of the trap, $\{\mathbf{R}\} \equiv\left\{\mathbf{R}_{1}, \mathbf{R}_{2}, \cdots, \mathbf{R}_{N_{L}}\right\}$ is a set of vectors describing the positions of the lattice potential minima on the $3 \times 3 \times 3$ cubic lattice cage considered, and $\alpha$ is a variational parameter signalling the strength of the external harmonic confinement. Essentially, $\alpha$ is the inverse overall width of the total wave function of the system in the CHOCL. Further, $\alpha$ controls the volume of the external harmonic confinement and, therefore, the number of lattice sites to be occupied away from the center of the trap. Eq. (5) is constructed in a manner similar to that of Jin et al. 33 in that we sum over a number of localized single-particle wave functions $\phi\left(\mathbf{r}_{i}, \mathbf{R}_{n}\right)$ centered in the optical lattice wells, each at $\mathbf{R}_{n} . \phi\left(\mathbf{r}_{i}, \mathbf{R}_{n}\right)$ is constructed similarly to the wave function used by $\mathrm{Li}$ et al. [7] and is given by:

$$
\begin{aligned}
\phi\left(\mathbf{r}_{i}, \mathbf{R}_{n}\right)= & \exp \left[-\beta\left(\mathbf{r}_{i}-\mathbf{R}_{n}\right)^{2}\right] \times \\
& {\left[1+\gamma\left(x_{i}-X_{n}\right)^{2}-\sigma\left(x_{i}-X_{n}\right)^{4}\right] \times } \\
& {\left[1+\gamma\left(y_{i}-Y_{n}\right)^{2}-\sigma\left(y_{i}-Y_{n}\right)^{4}\right] \times } \\
& {\left[1+\gamma\left(z_{i}-Z_{n}\right)^{2}-\sigma\left(z_{i}-Z_{n}\right)^{4}\right], }
\end{aligned}
$$

where $\beta, \gamma$, and $\sigma$ are further variational parameters in addition to $\alpha$. The onsite repulsion is partly controlled by the local density $\left|\phi\left(\mathbf{r}_{i}, \mathbf{R}_{n}\right)\right|^{2}$ via the variational parameter $\beta$ in Eq. (6), which confines the particles at each lattice site $(n)$. The interactions in the trial wave function are taken into account by the usual HS Jastrow function [21, 22]:

$$
f\left(r_{i j}\right)=\left\{\begin{array}{rll}
0 & : & r_{i j} \leq a_{c} \\
1-\frac{a_{c}}{r_{i j}} & : & r_{i j}>a_{c}
\end{array},\right.
$$

where $r_{i j}$ is the distance between a pair of bosons.

The VMC wave function (4) is optimized by minimizing the average energy with Powell's technique 46] employed in a previous publication [47. The ground state configuration is achieved when all the particles are distributed symmetrically in the spherical coordination shells around the center of the trap, and the overall boson-boson repulsion is minimal. Once the trial function Eq. (4) has been optimized, it is plugged into the VPI code as a starting wave function. The same properties are then evaluated as those using VMC.

\section{Tunneling}

Since we consider in this work only the strongly interacting regime, single-particle tunneling is very much suppressed in our systems. Hence, the atom-pair tunneling is dominant and is included in the overlap of the wave functions in neighboring wells. A qualitative measure for the overlap of the wave functions is evaluated, for simplicity, from trial-density functions fitted to slices of the integrated optical densities along one axis. For this purpose, the trial-density functions are similar to the one-dimensional version of Eq. (6) with an additional amplitude factor $A_{n}$, that is

$$
\begin{aligned}
& \phi_{f i t}\left(x, X_{n}\right)^{2}=A_{n} \exp \left[-\beta_{n}\left(x-X_{n}\right)^{2}\right] \times \\
& \left|1+\gamma_{n}\left(x-X_{n}\right)^{2}+\sigma_{n}\left(x-X_{n}\right)^{4}\right| .
\end{aligned}
$$

The absolute value is considered to make sure we do not run into negative densities. An example of such a fit is shown in Fig. 1 below. Here, the fitting function is:

$$
F\left(x ; X_{1}, X_{2}, X_{3}\right)=\sum_{n=1}^{3} \phi_{f i t}\left(x, X_{n}\right)^{2}
$$




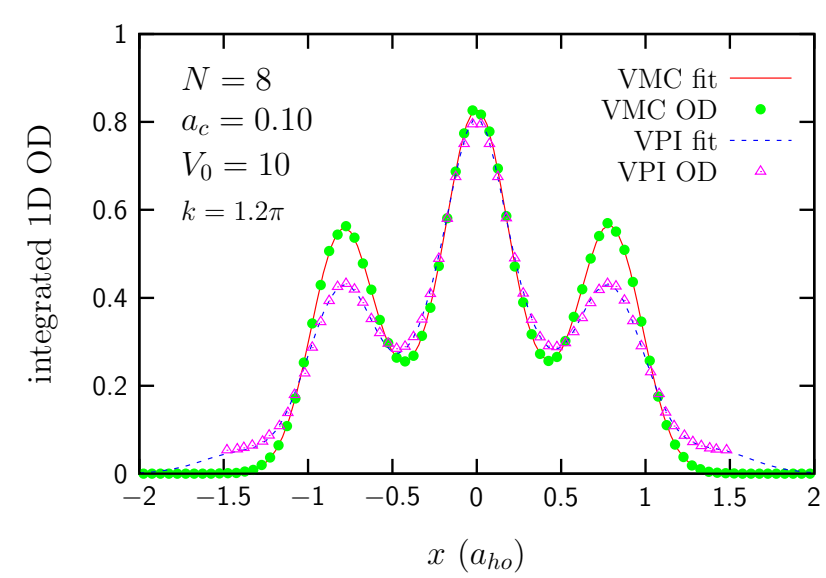

FIG. 1: Fits using the forms (8) and 9 to 1D VMC and VPI integrated optical density slices along the $\mathrm{x}$-axis for a system of $N=8, V_{0}=10$, and $k=1.2 \pi$. The sum of squares for both fits is $3.41 \times 10^{-4}$ for VMC and $7.41 \times 10^{-4}$ for VPI, respectively.

having peaks centered at three potential minima $X_{1}$ (left), $X_{2}$ (center), and $X_{3}$ (right) and with three sets of fitting parameters $A_{n}, \beta_{n}, \gamma_{n}, \sigma_{n}$, and the latter $X_{n}$, where $n$ runs from 1 to 3 . In fact, the central position $X_{2}$ always turns out to be exactly zero as required. The sum of squares

$$
\chi^{2}=\sum_{i=1}^{P}\left\{\sum_{n=1}^{3}\left(\left|\phi_{f i t}\left(x_{i}, X_{n}\right)\right|^{2}-\left|\phi_{M C}\left(x_{i}, X_{n}\right)\right|^{2}\right)\right\}^{2}
$$

is minimized with respect to the above sets of fitting parameters. Here $\chi^{2}$ is a sum over all $P$ data points of the one-dimensional integrated $\mathrm{MC}$ optical density $\left[\sum_{n=1}^{3}\left|\phi_{M C}\left(x_{i}, X_{n}\right)\right|^{2}\right]$. By this minimization, we get values of $\chi^{2}<10^{-3}$ indicating a good fit. After optimization, the overlap integral

$$
\begin{aligned}
& I_{\text {overlap }}=\int_{-\infty}^{+\infty} \phi_{f i t}\left(x, X_{1}\right) \phi_{f i t}\left(x, X_{2}\right) d x+ \\
& \int_{-\infty}^{+\infty} \phi_{f i t}\left(x, X_{2}\right) \phi_{f i t}\left(x, X_{3}\right) d x
\end{aligned}
$$

is then evaluated using a simple elementary numerical technique.

We must emphasize, that it is hard to describe the single-particle tunneling amplitude for strongly interacting systems using the well-known exchange integral [7,

$$
J_{n}=\int d^{3} r \phi_{n}(\mathbf{r})\left[-\frac{\hbar^{2}}{2 m} \nabla_{i}^{2}+V(\mathbf{r})\right] \phi_{n+1}(\mathbf{r})
$$

where $\phi_{n}$ and $\phi_{n+1}$ are single-particle wave functions at sites $n$ and $n+1$, and $V(\mathbf{r})$ a single-particle potential.
This is because the interactions as described by the Jastrow factor [Eq. (7)] are not included in Eq. (12). In fact, it is anticipated that the single-particle wave function narrows as the HS repulsion rises, counteracting the effects of the broadening due to the Jastrow factor as the number of particles is increased beyond a certain limit.

\section{Bosons-optical lattice overlap}

A measure for the extent of the overlap between $\phi\left(\mathbf{r}_{i}, \mathbf{R}_{n}\right)$ and $V_{\text {opt }}\left(\mathbf{r}_{i}\right)$ for all particles $i=1$ to $N$ and all lattice sites $n=1$ to $N_{L}$ is given by

$$
\left\langle I_{B O S O N-O L}\right\rangle=\sum_{n=1}^{N_{L}} \int_{\langle M C\rangle} e^{-\alpha r^{2}} \phi\left(\mathbf{r}, \mathbf{R}_{n}\right) V_{o p t}(\mathbf{r}) d^{3} r,
$$

where $\int_{\langle M C\rangle}$ stands for a MC configurational integral defined in Sec $\mathrm{IIH}$ below, and $O L$ stands for optical lattice. Further $\left\langle I_{B O S O N-O L}\right\rangle$ is an indirect measure of the total tunneling amplitude of the system into the optical lattice potential barriers.

\section{E. Discrete onsite interaction energies}

In order to explore the single-particle response to the change in the HS repulsions, we evaluate the onsite interaction energies at individual lattice sites. The onsite interaction energy for a single particle at each lattice site $(n)$ is evaluated using [7, 48,

$$
\left\langle U_{n}\right\rangle=g \int_{\langle M C\rangle} e^{-4 \alpha r^{2}}\left|\phi\left(\mathbf{r}, \mathbf{R}_{n}\right)\right|^{4} d^{3} r,
$$

where $g=4 \pi \hbar^{2} a_{c} / m$ with $a_{s}$ replaced by $a_{c}$. Here the effects of the external trap are included via the factor $\left[\exp \left(-\alpha r^{2}\right)\right]^{4}$.

\section{F. Occupancy of Lattice Sites}

Another single-particle response is the atom-number occupancy of each lattice site. The average number of particles occupying a particular lattice site $(n)$ is evaluated via

$$
\left\langle N_{n}\right\rangle=N \int_{\langle M C\rangle} e^{-2 \alpha r^{2}}\left|\phi\left(\mathbf{r}, \mathbf{R}_{n}\right)\right|^{2} d^{3} r .
$$

An alternative way to determine the occupancy of each lattice site, is to divide the confining volume into equivalent cubic bins whose corners are sets of four lattice sites $\mathbf{R}_{n}$ and count the number of particles collected in each bin. We used this counting method to evaluate the VPI $\left\langle N_{n}\right\rangle$, whereas 15 to obtain the $\operatorname{VMC}\left\langle N_{n}\right\rangle$. 


\section{G. Correlations between the bosons}

The pair correlation function $g(r)$ is evaluated using VPI by binning pairs of particles in each $r$. The idea is to provide a measure for the strength of the bosonboson correlations and its dependency on the repulsive interactions in a tightly confining environment.

\section{H. Numerics}

The integrals (13), 14 and (15) are evaluated using standard Monte Carlo integration [45]:

$\int_{\langle M C\rangle} O\left(\mathbf{r}_{i}\right) d \mathbf{r}_{i}=\frac{1}{N} \sum_{i=1}^{N} \frac{1}{M} \sum_{c=1}^{M} \frac{O\left(\mathbf{r}_{c i}\right)}{e^{-2 \alpha r_{c i}^{2}} \sum_{n=1}^{N_{L}}\left|\phi\left(\mathbf{r}_{c i}, \mathbf{R}_{n}\right)\right|^{2}}$

where $c$ is a configurational index, $i$ a particle index, and $M$ is the total number of VMC configurations. We further sum over all $N$ particles and divide by $N$ to get the average. The denominator is a weight we used for all MC integrals. In VPI we apply the same integration technique, except with $M$ taken as the total number of time slices used in the path integral. Each time slice contains one configuration of $N$ particle positions.

\section{Computational complexities}

As much as VPI is an accurate method for the present determination of the properties of lattice bosons, using a number of particles exceeding $N=8$, this method begins to constitute a heavy-computational and CPU-time consuming technique. Particularly, for large optical potential barriers $V_{0} \geq 10 \hbar \omega_{h o}$, the bosons take a very long CPU time to tunnel through the barriers in order to achieve a symmetric optical density distribution inside the whole trap. We therefore limited the number of particles to $N=8$ when using VPI. On the other hand, the less accurate VMC method allows the bosons to diffuse quickly through the barriers providing a computationally cheap and fast technique to investigate qualitatively the properties of bosons in optical lattices. Our aim is, however, not to present methods but to concentrate on the physics of quantum tunneling.

\section{RESULTS}

In what follows, we present the results of our calculations. We particularly focus on the effect of interactions on the tunneling amplitude $\propto I_{\text {overlap }}$. We further explore the effect of $a_{c}$ on the onsite interaction energies $\left\langle U_{n}\right\rangle$, energy per particle $\langle E\rangle / N$, the occupancy of lattice sites $\left\langle N_{n}\right\rangle$, the correlations $g(r)$, and the optical density. As mentioned before, we compare our VMC results with corresponding VPI results.

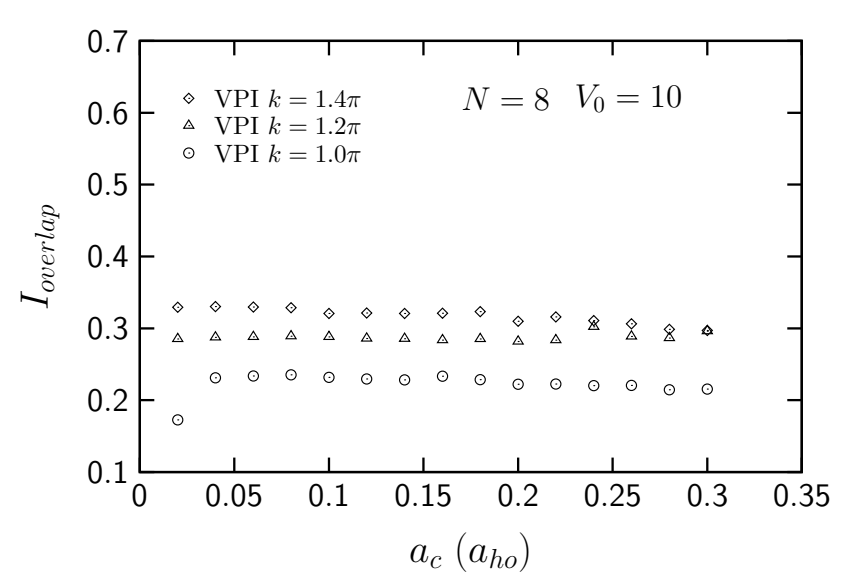

,FIG. 2: Tunneling amplitude $\propto I_{\text {overlap }}$ [Eq. [11] ] vs the hardsphere (HS) diameter $a_{c}$, obtained from fits to VPI optical densities. The system is a HS Bose gas of $N=8$ particles in a $3 \times 3 \times 3$ cubic optical lattice of depth $V_{0}=10$ embedded in a tight external harmonic trap of trapping frequency $\omega_{h o}$. Open circles: $k=\pi$, open triangles: $k=1.2 \pi$, and open diamonds: $k=1.4 \pi$. Energies and lengths are in units of the trap.

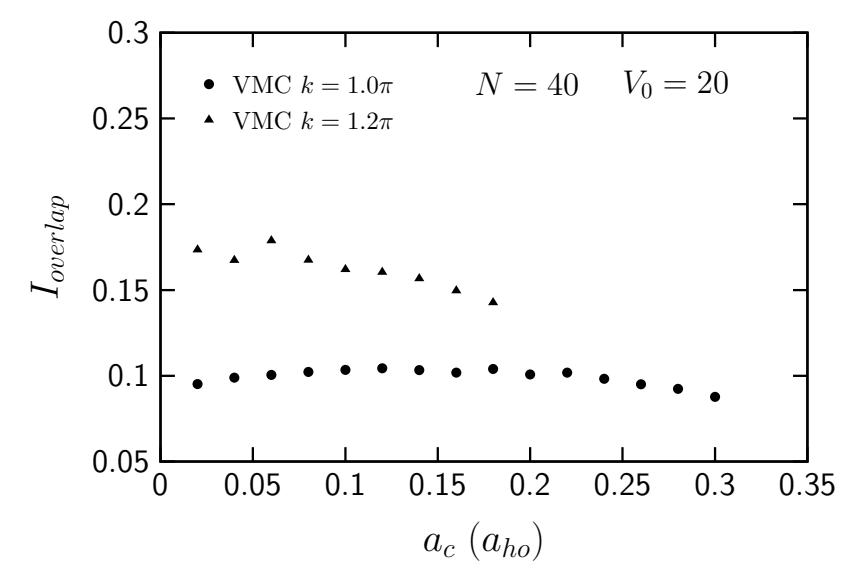

FIG. 3: As in Fig. 2, but using VMC for $N=40, V_{0}=20$ and the indicated values of $k$.

\section{A. Tunneling}

$I_{\text {overlap }}$ is now displayed as a function of $a_{c}$. Fig. 2 displays the VPI results for systems of $N=8$ and $V_{0}=10$ with $k=\pi$ (open circles), $k=1.2 \pi$ (open triangles) and $k=1.4 \pi$ (open diamonds). For these values of $N$ and $V_{0}, I_{\text {overlap }}$ shows a very weak response to the effects of increasing $a_{c}$, since it seems to remain practically constant. As $k$ is increased, $I_{\text {overlap }}$ rises indicating a profound effect of the lattice spacing $d=\pi / k$ on the tunneling amplitude.

The case for $N=40$ and $V_{0}=20$ is shown in Fig. 3 for $k$-values of $\pi$ and $1.2 \pi$ (solid circles and triangles, respectively). For $k=1.2 \pi$, $I_{\text {overlap }}$ decreases overall 


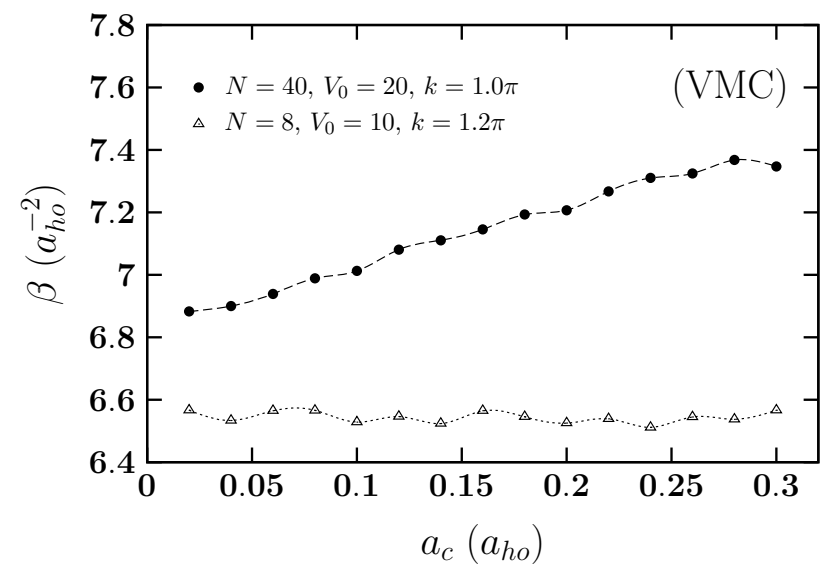

FIG. 4: VMC parameter $\beta$ of the trial wave function [Eq. [6] ] as a function of the hard-sphere diameter $a_{c}$ for two HS Bose gas systems in a combined harmonic simple cubic optical lattice of $3 \times 3 \times 3$ sites. Solid circles: HS Bose gas of Fig. 2 at $k=1.2 \pi$. Open triangles: HS Bose gas of Fig. 3 at $k=\pi$.

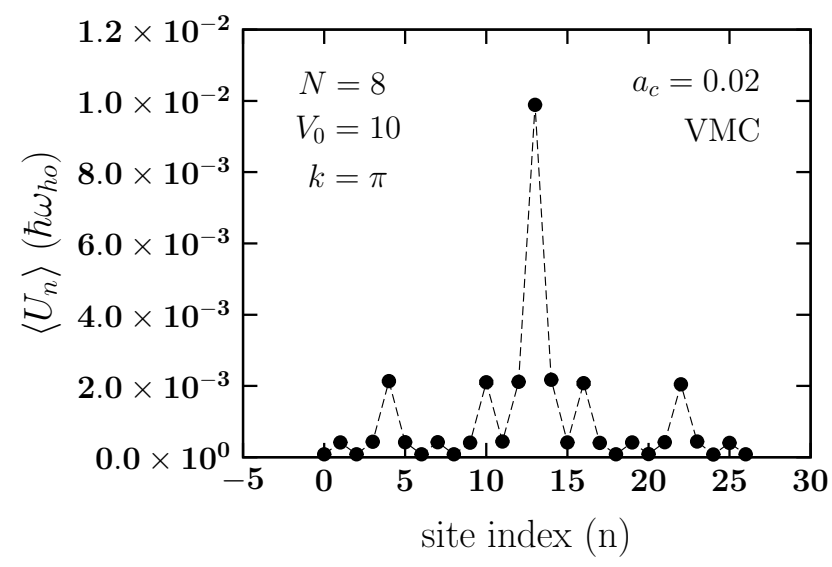

FIG. 5: Average VMC onsite repulsive interaction energy $\left\langle U_{n}\right\rangle$ at each lattice site $n$ for a HS Bose gas of $N=8$ particles, optical lattice depth $V_{0}$, and HS diameter $a_{c}=0.02$ in a $3 \times 3 \times 3$ cubic optical lattice embedded in a tight external harmonic trap of trapping frequency $\omega_{h o}$. Energies and lengths are in units of the trap.

with increasing $a_{c}$, whereas for $k=1.0 \pi$ it rises somewhat up to $a_{c}=0.18$ and then begins to drop. However, the response is still weak and insignificant. Hence, a slightly stronger response is obtained for the tunneling amplitude when using a larger $N$. Further, the effect of changing $d=\pi / k$ on $I_{\text {overlap }}$ is much more pronounced than changing $a_{c}$. Signals for a stronger response of our systems to the rise of $a_{c}$ are found in the single-particle properties such as the width of the single-particle wave function and the onsite interaction energy.

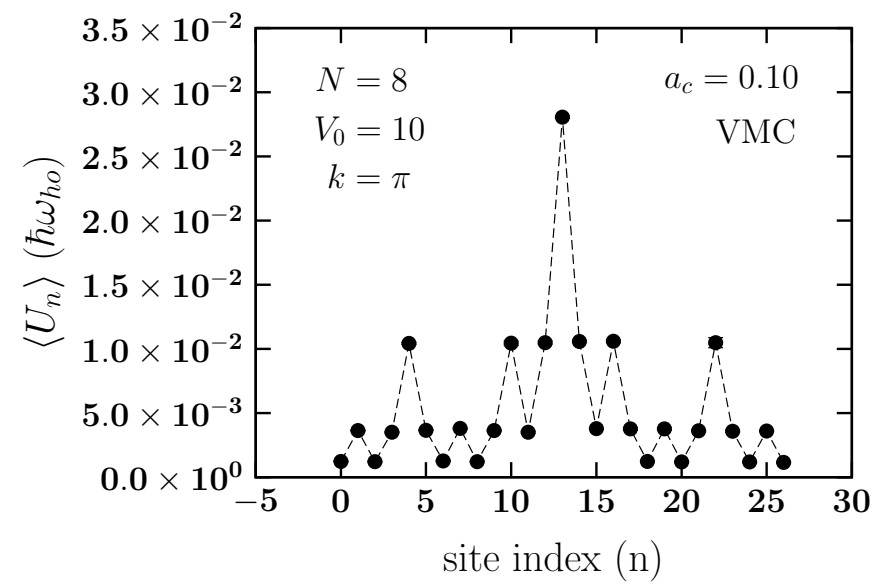

FIG. 6: As in Fig. 5 but for $a_{c}=0.10$.

\section{B. Width of the single-particle wave function in each potential well}

As already indicated in the Method section, the width of the single-particle wave function $\phi\left(\mathbf{r}, \mathbf{R}_{n}\right)$ [Eq.(6)] in each well, is expected to decrease with a rise of the HS repulsion in order to counteract the effects of a broadening due to the Jastrow function for a larger number of particles. The width, or better the inverse of it, is described by the VMC parameter $\beta$ in Eq. 6). In Fig. 4, the VMC parameter $\beta$ of the local wave function, $\phi\left(\mathbf{r}, \mathbf{R}_{n}\right)$, is displayed as a function of $a_{c}$. The open triangles display $\beta$ for the HS Bose gas of Fig. 2 at $k=1.2 \pi$; solid circles: HS Bose gas of Fig. 3 at $k=\pi$. It is found that this width $(\propto 1 / \sqrt{\beta})$ decreases with the rise in the repulsion for $N=40$ and remains almost constant for $N=8$.

\section{Onsite interaction energies}

Discrete distributions of $\left\langle U_{n}\right\rangle$ over all 27 lattice sites, for systems of $N=8, V_{0}=10, k=\pi$, and two cases $a_{c}=0.02$ and 0.10, are displayed in Figs. 5 and 6, respectively. The abcissa display the site index $n$. All $\left\langle U_{n}\right\rangle$ which have the same magnitude within a small margin of error, belong to the same set of nearest neighbors from the central lattice site. That is, moving vertically from the tip of the discrete distribution down to the bottom, we move from the center to the first, second, and third set of nearest neighbors, respectively. The distribution is uniform around the trap center. The effects of the external trap are manifested in the magnitude of $\left\langle U_{n}\right\rangle$ which is highest at the center and declines towards the edges of the trap due to the factor $\exp \left(-4 \alpha r^{2}\right)$ in Eq.(14). Obviously, a large percentage of the particles is concentrated at the central lattice site. (It is anticipated then, that if the strength of the external harmonic trap is increased further, this causes a drop in the density $\sim \exp \left(-2 \alpha r^{2}\right)\left|\phi\left(\mathbf{r}, \mathbf{R}_{n}\right)\right|^{2}$ at the lattice sites towards the 


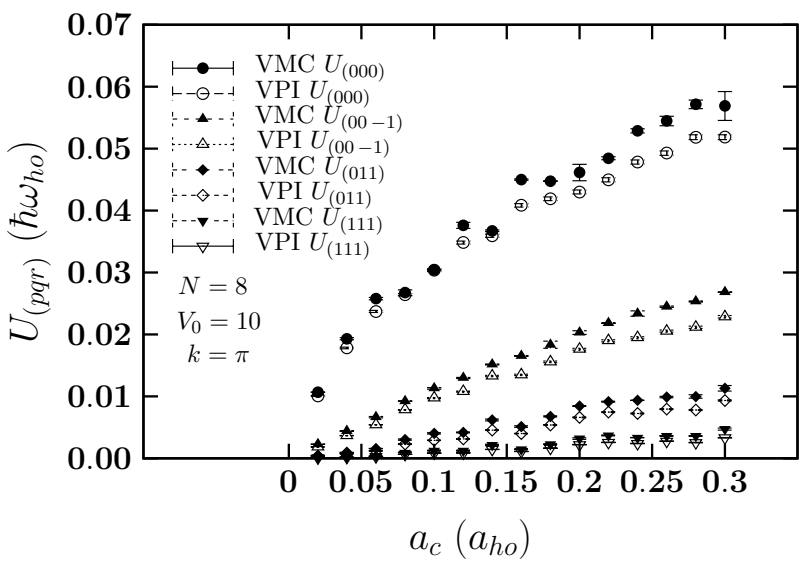

FIG. 7: Average onsite repulsive interaction energy $\left\langle U_{(p q r)}\right\rangle$ vs the hard-sphere (HS) diameter $a_{c}$ at four lattice sites representative of the whole lattice. The system is the HS Bose gas mentioned in Fig. 2 at $k=\pi$. Solid and open circles: VMC and VPI results for $\left\langle U_{(000)}\right\rangle$. Solid and open triangles: likewise for $(00-1)$. Solid and open diamonds: for $(011)$. Solid and open inverted triangles: for (111).

edges of the trap and a certain rise at the center of the trap.) Eventually, all the bosons will occupy the central lattice site and only if their HS diameter is of a magnitude that allows all of them them to fit inside one lattice-site volume $\sim 4 \pi(d / 2)^{3} / 3$. Thus, a large amount of repulsive potential energy would be stored in the trap center. The patterns of Figs. 5 and 6 show also that the bosons minimize their potential energy arising from the external harmonic trap by maximizing their occupancy of the trap center, and minimizing it towards the edges of the trap.

In addition, $\left\langle U_{n}\right\rangle$ is displayed as a function of $a_{c}$ in Figs. 7 and 8 for the systems of Fig. 2 at $k=\pi$ and $1.2 \pi$, respectively, and for four lattice sites: $\mathbf{R}_{n} \equiv(000)$, $(00-1),(111)$, and (011), representative of the whole lattice. In general, $\left\langle U_{(p q r)}\right\rangle$ rises with $a_{c}$ signaling a rise in the repulsive energy of the particles in each well. The rate of growth of $\left\langle U_{(p q r)}\right\rangle$ is largest for the trap center and declines towards the edges of the trap.

\section{Boson-optical lattice overlap}

In this section, we evaluate $\left\langle I_{B O S O N S-O L}\right\rangle$ [Eq. [13] ] which measures the overlap of all the single particle wave functions in the wells with the periodic optical lattice potential. In essence, it measures also the total tunneling amplitude of the particles into the optical lattice potential barriers. Figs. 9 and 10 display $\left\langle I_{B O S O N-O L}\right\rangle$ vs. $a_{c}$ for the systems of Figs. 2 and 3 respectively. One can see that $\left\langle I_{B O S O N-O L}\right\rangle$ is practically invariant for $N=8$, but the system with $N=40$ reveals a slightly stronger response to changes in $a_{c}$. For example for $N=8$ and $k=\pi$, the change in $\left\langle I_{B O S O N-O L}\right\rangle$ from $a_{c}=0.02$ to 0.3 is only by $-1.45 \%$. The other changes for $N=8$ are

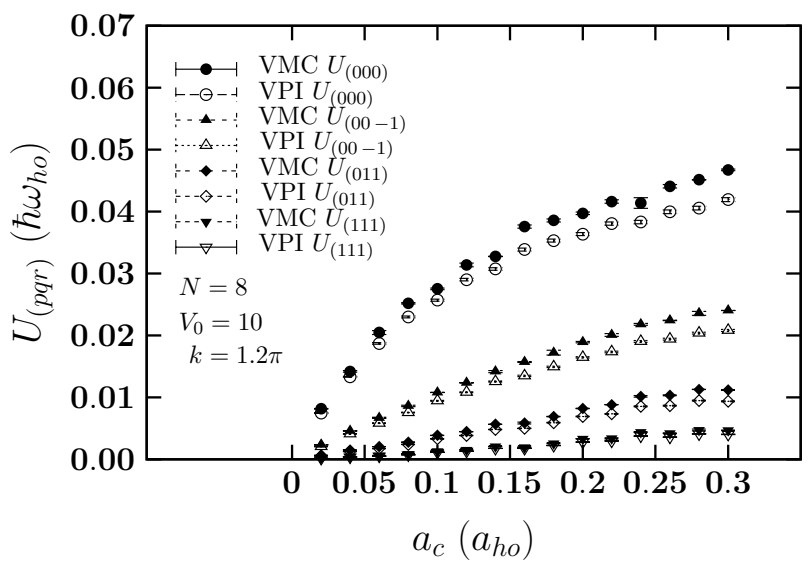

FIG. 8: As in Fig. 7. but for $k=1.2 \pi$.

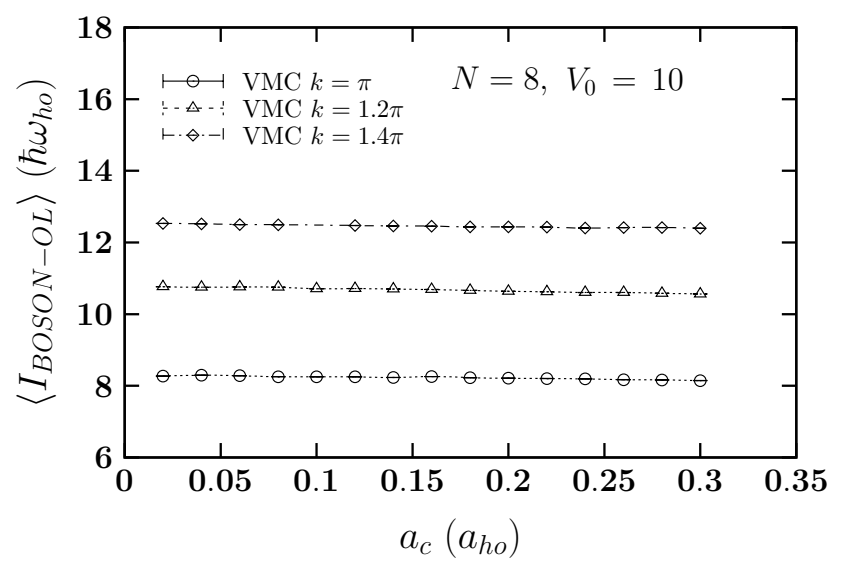

FIG. 9: VMC disorder integral [Eq. 13]] vs the hard-sphere diameter $a_{c}$ for the systems in Fig. 2 .

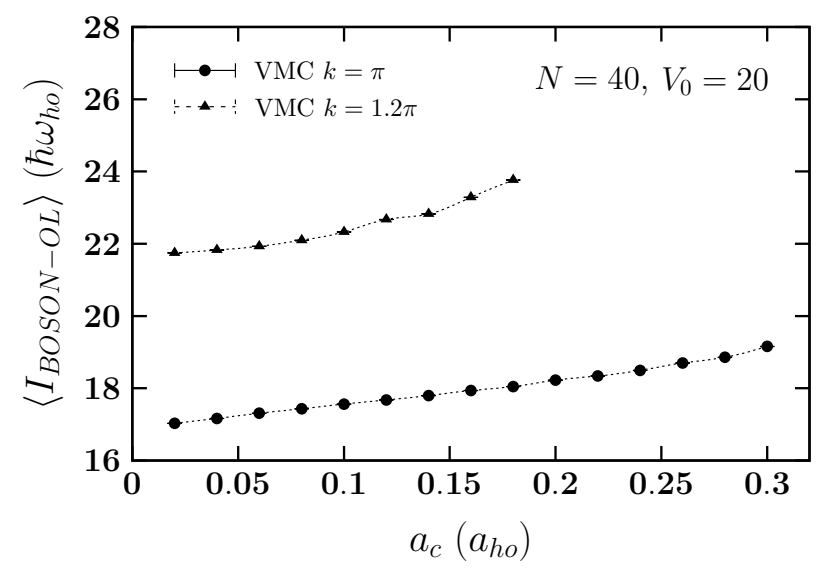

FIG. 10: VMC disorder integral [Eq. 13] $]$ vs the hard-sphere diameter $a_{c}$ for the systems in Fig. 3 .

of the same order of magnitude. This in turn indicates, that the width of each localized wave function for these 
systems is practically invariant with the rise in repulsion. This may come as a surprise, since it is known [21, 22. that at very large HS diameters such as the ones used here, the wave function ought to become very broad indeed. It seems that the localization effect of the optical lattice potential overwhelms the repulsive forces tending to broaden the wave function in each well.

One further observes that $\left\langle I_{B O S O N-O L}\right\rangle$ increases with $k$ in agreement with the results of Figs. 2 and 3 , where $I_{\text {overlap }}$ rises with increasing $k$.

Further, we note that in Fig. $10\left\langle I_{B O S O N-O L}\right\rangle$ rises notably with $a_{c}$ indicating a rise in the total tunneling amplitude into the optical potential barriers. We were unable to obtain values of $\left\langle I_{B O S O N-O L}\right\rangle$ for $k=1.2 \pi$ beyond $a_{c}=0.18$ as we could not find VMC energy minima for these systems. We must emphasize that the overlap of the bosons with the optical lattice potential is a different measure than $I_{\text {overlap }}$, which measures the overlap of two wave functions in neighboring wells.

\section{E. Occupancy of lattice sites}

Figure 11 displays the average occupancy of four lattice sites $\left\langle N_{(p q r)}\right\rangle$ for systems of Fig. 2 $(p q r) \equiv(000)$, $(00-1),(011)$, and (111). Each frame is labelled by the corresponding lattice site. The solid and open diamonds display VMC and VPI results for $k=1.4 \pi$, whereas the solid and open triangles those for $k=1.2 \pi$, respectively. The VMC results have been obtained by Eq. (15), whereas the VPI results from simple counting of the bosons in cubic bins. In frames $(000)$ and $(00-1)$, the occupancy decreases with $a_{c}$, whereas in frames (011) and (111) it rises. Further, the occupancy is higher for a lower $k$, i.e., a larger lattice volume. The VMC and VPI results almost match for $(000)$ and $(00-1)$ and are close to each other for (011) and (111). There is good agreement between the occupancies calculated by Eq.(15) and those obtained by counting. This indicates that the single-particle wave function in Eq. (6) is suitable to describe the bosons at each lattice site.

\section{F. Correlations}

The VPI pair correlation functions $g(r)$ for the systems of $N=8, V_{0}=10, k=\pi$, and various $a_{c}$ in the range $0.02 \leq a_{c} \leq 0.30$ are displayed in Fig. 12 . It is observed that the correlations are strongest for $a_{c}=0.02$ and gradually weaken as $a_{c}$ is increased.

\section{G. Energy}

The average Monte Carlo (MC) energies per particle $\langle E\rangle / N$ as functions of $a_{c}$ for the systems of Figs. 2 and 3, are displayed in Figs. 13, and 14, respectively. Additionally, the energies for a system of $N=2$ and

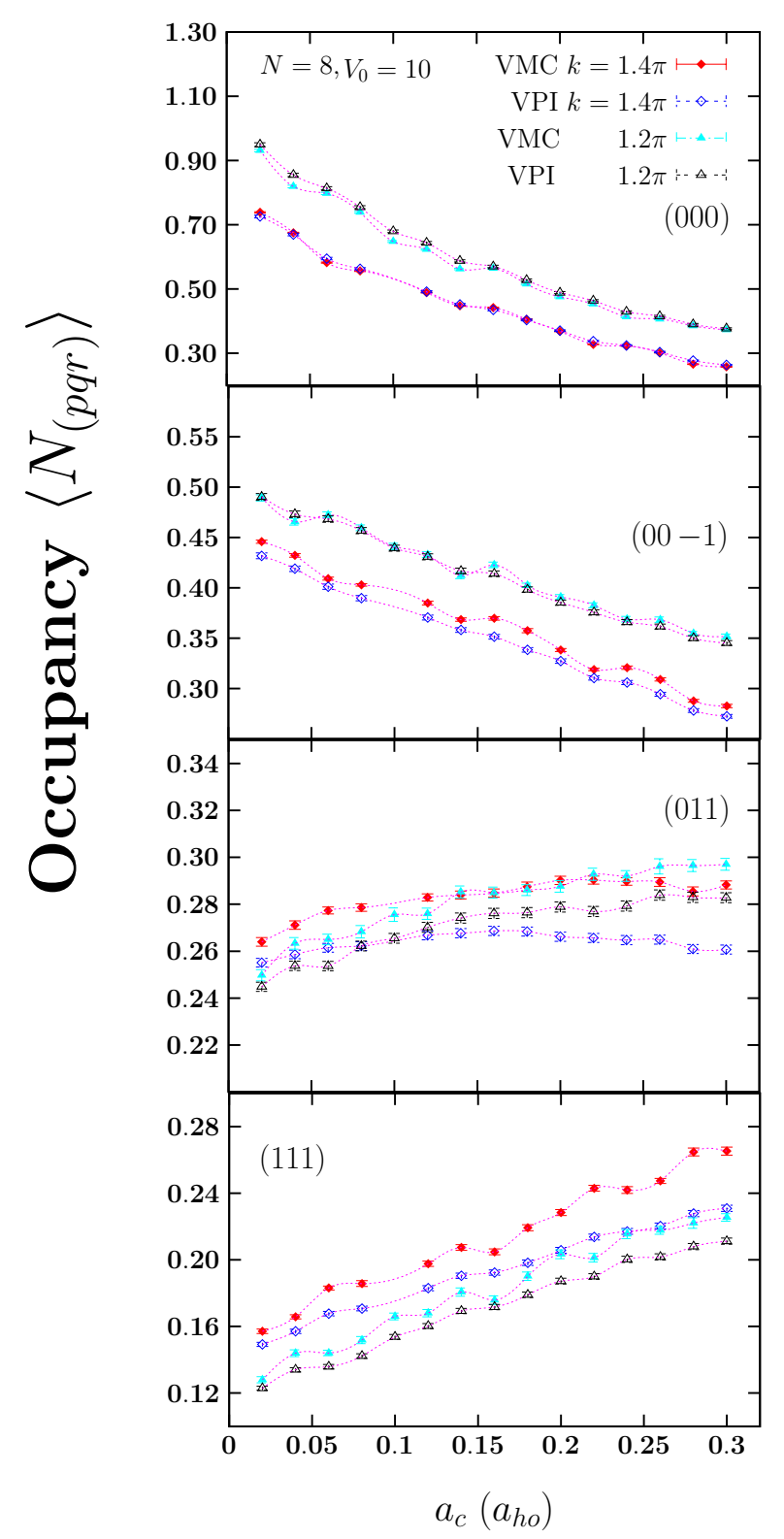

FIG. 11: (Color online) Average occupancy $\left\langle N_{(p q r)}\right\rangle$ vs the hard-sphere diameter $a_{c}$ at the four lattice sites $(p q r)$ indicated, which are representative of the whole optical lattice. The system is the HS Bose gas of Fig. 2. Solid and open diamonds: VMC and VPI results for $k=1.4 \pi$, respectively. Solid and open triangles: likewise, but for $k=1.2 \pi$. From top to bottom frame: $\mathbf{R}_{13} \equiv(000)$ is the center, $\mathbf{R}_{12} \equiv(00-1)$ the first, $\mathbf{R}_{17} \equiv(011)$ the second, and $\mathbf{R}_{26} \equiv$ (111) the third nearest neighbor to the center, respectively. Site indices $n$ are according to Fig. 5

$V_{0}=2$, are displayed in Fig. 15 as well. The same legends are used in the latter three figures as follows. Solid and open diamonds: VMC and VPI results for $k=1.4 \pi$, respectively. Similarly for triangles and circles: $k=1.2 \pi$ and $\pi$, respectively. We note that, for 


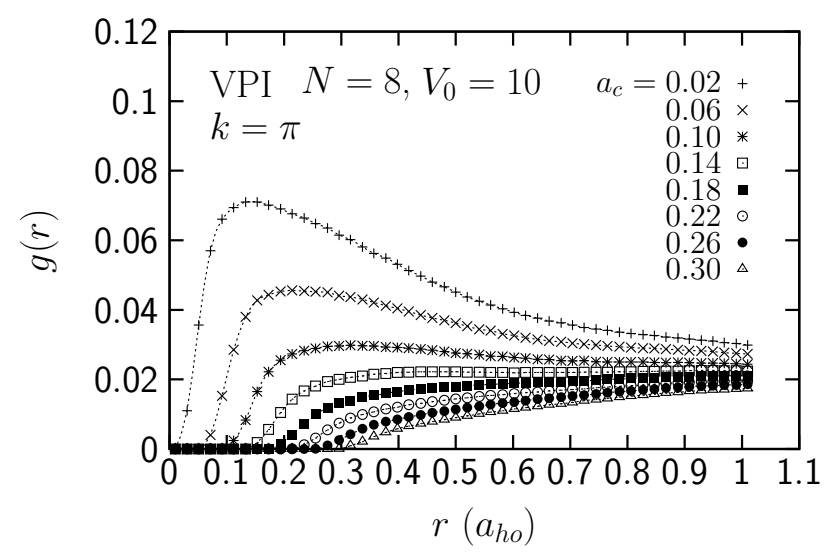

FIG. 12: VPI pair correlation function $g(r)$ for the indicated $a_{c}$. The system is the HS Bose gas of Fig. 2 at $k=\pi$.

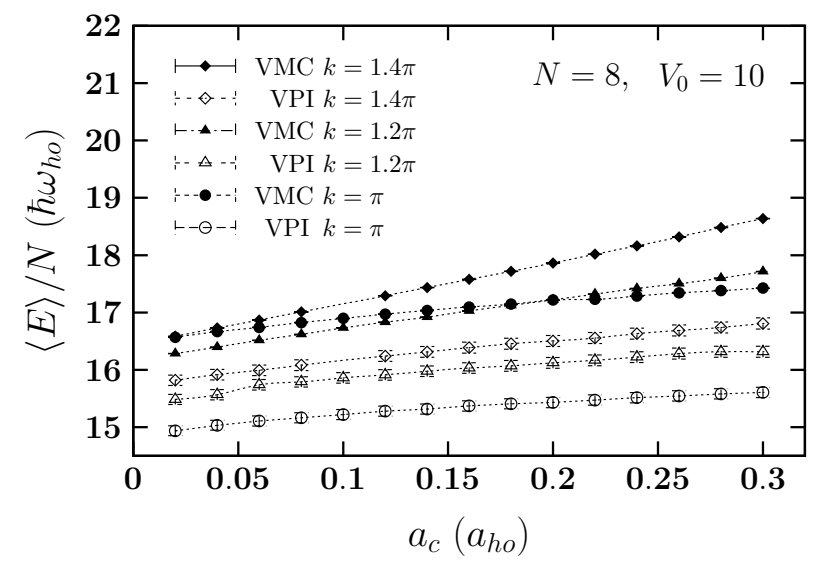

FIG. 13: Average energy per particle $\langle E\rangle / N$ vs. $a_{c}$ for a HS Bose gas system of $N=8, V_{0}=10$, and the indicated values of $k$. Solid and open diamonds: VMC and VPI results for $k=1.4 \pi$. Solid and open triangles: likewise, but for $k=1.2 \pi$, and solid and open circles: $k=\pi$.

most of the systems considered, $\langle E\rangle / N$ changes almost linearly within the given range of $a_{c}$ at a much slower rate than HS Bose gases in pure harmonic traps 21, 22. For example for $N=2$ with $k=\pi$, the $\operatorname{VPI}\langle E\rangle / N$ rises only by $\Delta E_{V P I} / N \sim+2.98 \%$ from $a_{c}=0.02$ to $a_{c}=0.3$, despite the fact that there is a very large change in $a_{c}$ (by a factor of 14!). Similarly for $N=8$ and $k=\pi, \Delta E_{V P I} / N$ is $\sim+4.48 \%$. However, $\Delta E_{V P I} / N$ for $k=1.4 \pi$ is more pronounced, being $\sim+6.26 \%$. The corresponding VMC results are as follows: for $N=2$ with $k=\pi, \Delta E_{V M C} / N \sim+4.48 \%$, for $N=8$ with $k=\pi, \Delta E_{V M C} / N \sim+5.20 \%$, and for $N=8$ with $k=1.4 \pi, \Delta E_{V M C} / N \sim+12.37 \%$, respectively. For $N=40, \Delta E / N$ changes at a much faster rate than for a lower $N$. Further, the energy $\left\langle E_{V M C}\right\rangle / N$ rises with increasing $k$.

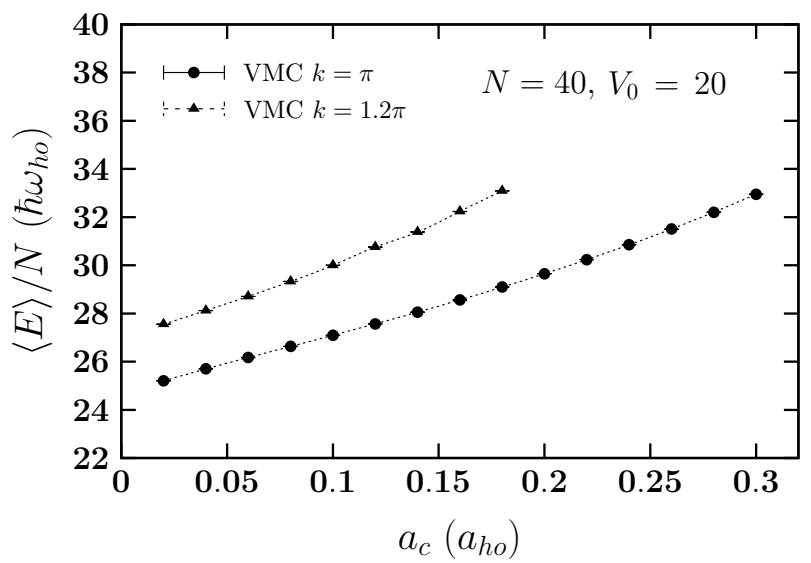

FIG. 14: As in Fig. 13, but for the HS Bose gases of Fig. 3

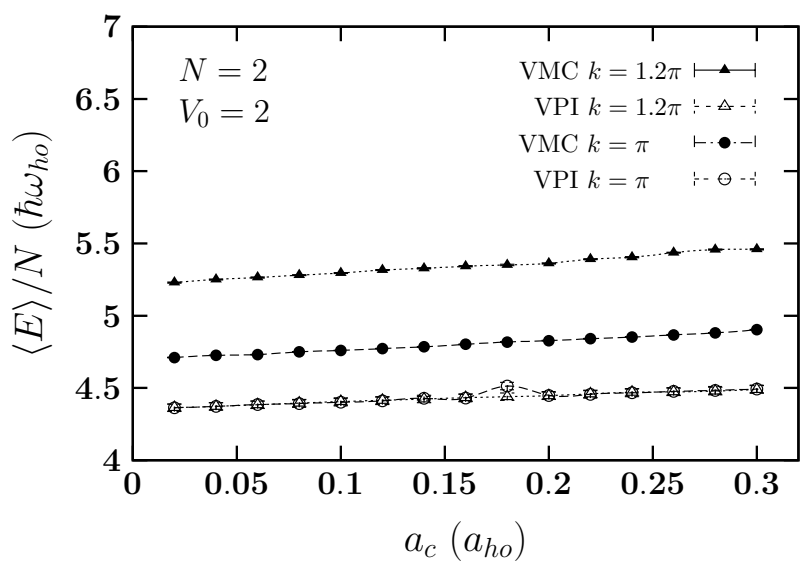

FIG. 15: As in Fig. 13 but for $N=2, V_{0}=2$, and the indicated values of $k$.

\section{H. Optical density}

In this section, the integrated VPI optical density (OD) is displayed at different $a_{c}$. The goal is to explore the effects of variations in the interactions on the optical density profiles and on the tunneling amplitude. Particularly we show visually that, for a small number of particles, the width of the wave function in each well does not broaden with an increase in $a_{c}$ even up to order 0.1 and that the overlap between the wave functions does not change either.

Fig. 16 displays the integrated VPI OD for $N=2$, $V_{0}=2$, and $k=\pi$. From the top to bottom frames, $a_{c}=0.02,0.14$, and 0.30, respectively. As expected, the amplitude of the density is maximal at the center of the trap and lowest for the lattice sites near the edges of the trap. Further, the tunneling of the system into the potential barrier of the external harmonic trap is suppressed due to the steep rise of this barrier as one goes away from the center of the trap. Since the optical depth is very shallow, the wave function of the system is spread 

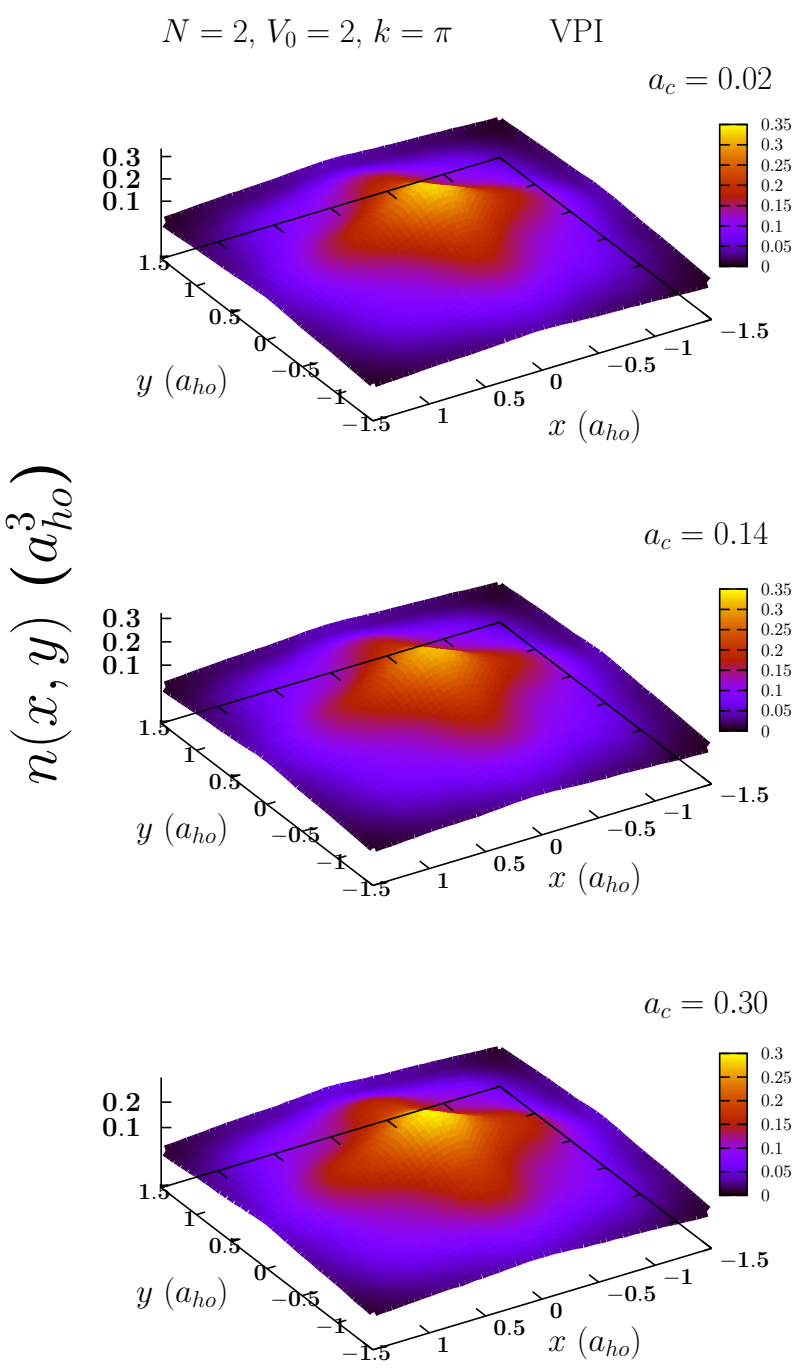

FIG. 16: Integrated VPI optical density for the HS Bose gas system of $N=2, V_{0}=2$, and $k=\pi$, at three values of $a_{c}$. From top to bottom frames: $a_{c}=0.02,0.14$, and 0.30, respectively. Density and lengths are in trap units.

over the whole lattice, indicating the dominance of the tunneling process [1] over the localization effects of the optical lattice potential wells. Superflow in these systems is thus prevailent.

Fig. 17 displays one-dimensional density slices of Fig. 16 along the $\mathrm{x}$-axis, and additionally three functions of the form (8) fitted to this density profile. The fits reveal the extent of the overlap between the wave functions in neighboring wells. It is observed, that this overlap does not change with a rise in $a_{c}$. The left (dashed line) and right (dotted line) density functions are much broader than the central function (thin dashed line) and all three peaks have almost the same amplitude. In fact, the overlap here is between three wave functions as those at the edges are able to spread over several trap lengths.

Fig. 18 is the same as Fig. 16, but for $N=8$ and

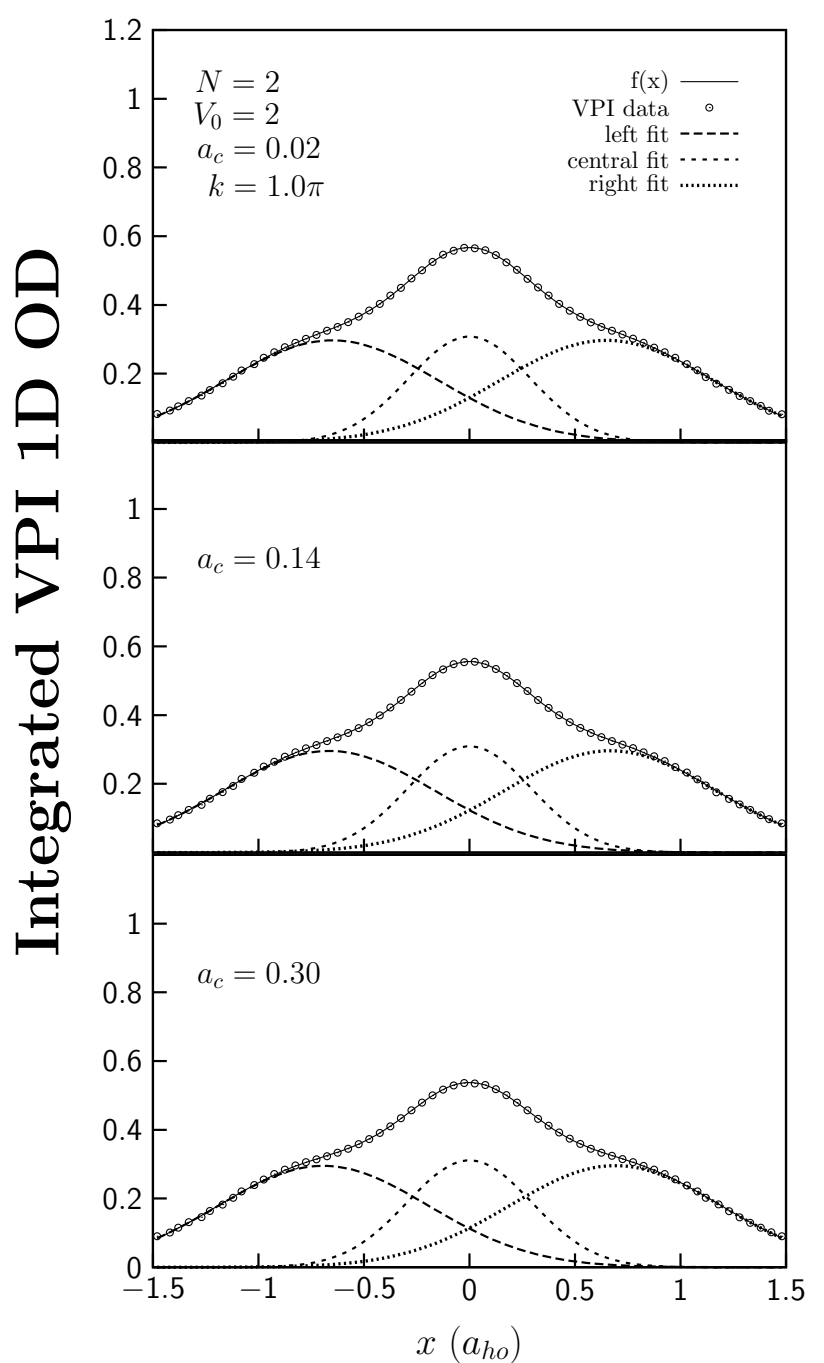

FIG. 17: Integrated one-dimensional optical density slices of Fig. 16 along the x-axis. Solid line: fitting function $f(x)=F\left(x ; X_{1}, X_{2}, X_{3}\right)$ Eq. 9 , open circles: VPI data, thick dashed line: left fit $\left|\phi_{f i t}\left(x, X_{1}\right)\right|^{2}$, thin dashed line: central fit $\left|\phi_{f i t}\left(x, X_{2}=0\right)\right|^{2}$, dotted line: right fit $\left|\phi_{f i t}\left(x, X_{2}\right)\right|^{2}$. From top to bottom, same $a_{c}$ at in Fig. 16 .

$V_{0}=10$. Here, the bosons are more localized at the lattice sites, and the tunneling process is less dominant than the localization effects. Again, the effects of the external trap are manifested: the density diminishes towards the edges of the trap. Fig. 19 displays again the one-dimensional slices of Fig. 18 along the x-axis. Here, the overlap is much less pronounced than in Fig. 17, and the amplitude of the central peak is larger than the peaks at the edges. The left and right peaks do not overlap as in Fig. 17. It seems that a slight increase in the number of particles has a profound effect on the density profiles in that it changes the ratio of the amplitude of the central density to that of the density at the trap edges. Further, the width of the densities at the edges of the trap for $N=8$ has dropped by almost 2 trap lengths as 


$$
N=8, V_{0}=10, k=\pi \quad \text { VPI }
$$

$$
a_{c}=0.02
$$

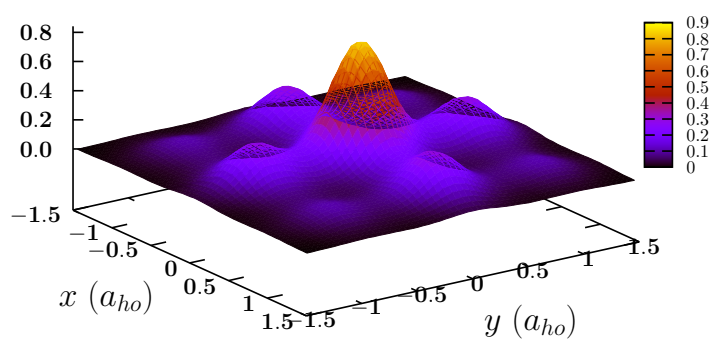



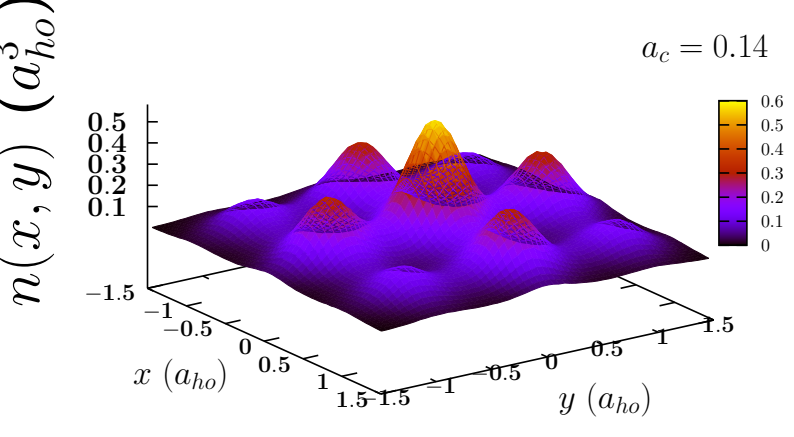

$a_{c}=0.30$

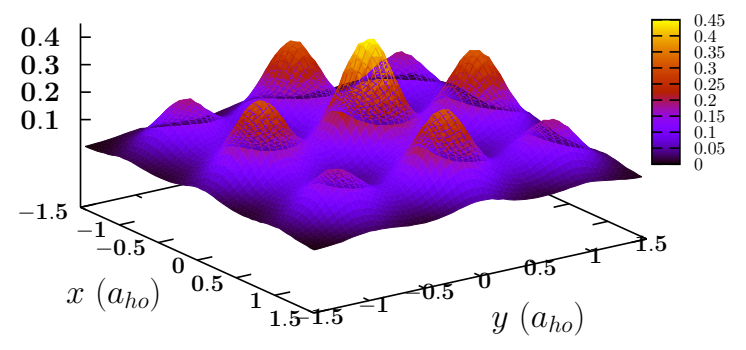

FIG. 18: As in Fig. 16 but for $N=8$ and $V_{0}=10$.

compared to $N=2$. Nevertheless, there is still overlap between the wave functions in the neighboring wells even at high repulsion. For $N=8$, the amplitude of the central peak declines with increasing $a_{c}$. The change in the height of the central peak as one increases $a_{c}$ from 0.02 to 0.14 is $\sim 33.3 \%$. Remarkably, even with strongly repulsive bosons, the density of the system in each well largely peaks at the potential minimum of each well instead of being broader and more evenly distributed around each lattice site as in Fig. 16.

The latter situation does not change very much for a larger number of particles. Fig. 20 displays the integrated optical density for the system of $N=40, V_{0}=20$, and $k=1.2 \pi$ and Fig. 21 its density slices, as in the latter figures for the optical density. Again, the peaks in each well do not broaden very much with a rise in $a_{c}$. The central peak looses amplitude in favor of the peaks at the edges. On closely inspecting the area of the overlap between neighboring peaks, one can depict a small decline in the

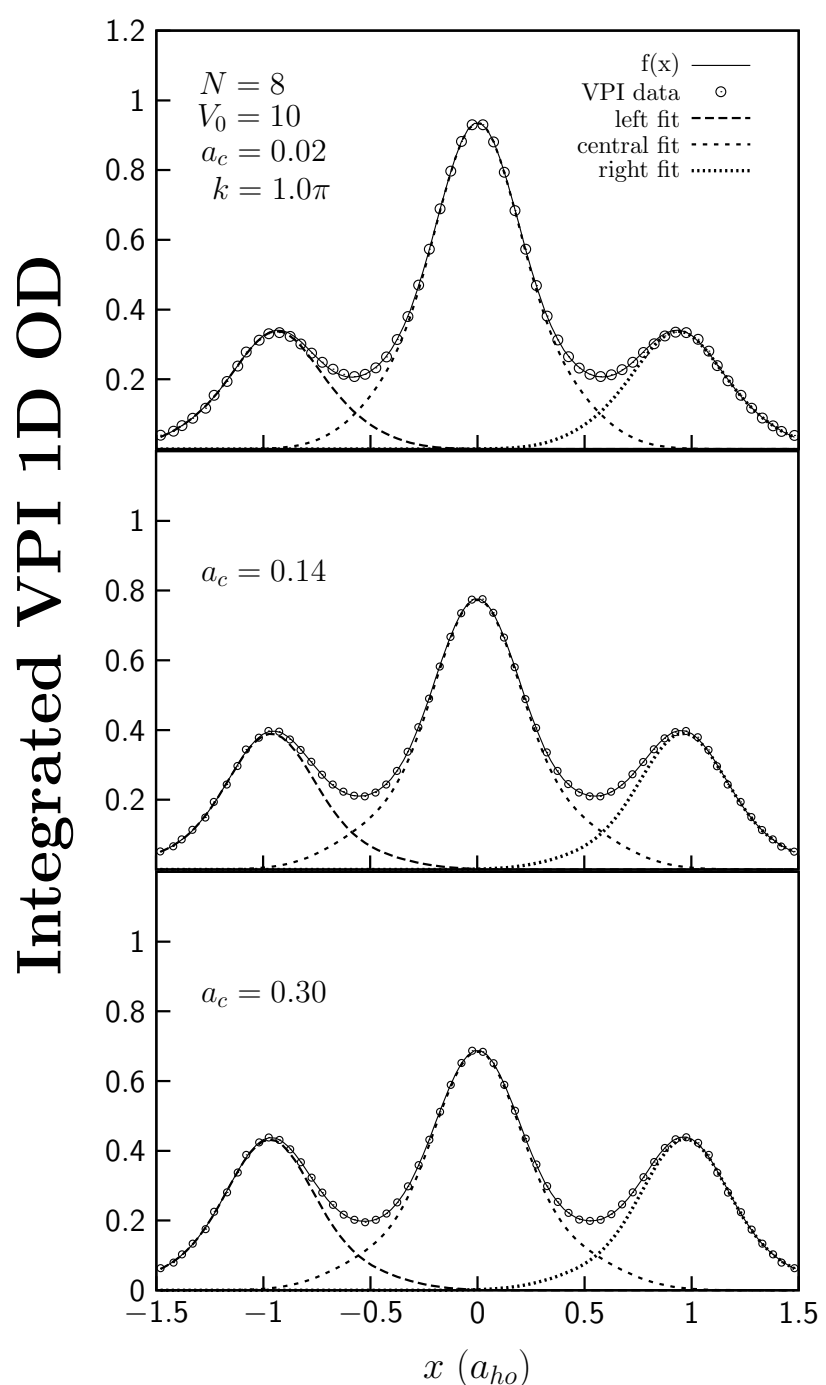

FIG. 19: As in Fig. 17 but for $N=8$ and $V_{0}=10$.

tunneling amplitude of the system as $a_{c}$ is increased. In Figs. 19 and 21, the positions of the left and right peak maxima shift to the left and right, respectively, as $a_{c}$ is increased.

\section{Alternative VMC Trial Function}

In order to test the validity of using a Gaussian $\left(\exp \left(-\alpha r^{2}\right)\right)$ in our trial wave function Eq. (4), we calculated the optical densities for $N=8, V_{0}=10$, and $k=\pi$ using a slightly modified version of our trial wave function:

$$
\begin{aligned}
& \Psi(\{\mathbf{r}\},\{\mathbf{R}\})= \\
& \prod_{i=1}^{N} \exp \left(-\alpha r_{i}^{2}-\epsilon r_{i}^{4}\right) \psi\left(\mathbf{r}_{i},\{\mathbf{R}\}\right) \prod_{i<j} f\left(\left|\mathbf{r}_{i}-\mathbf{r}_{j}\right|\right),
\end{aligned}
$$




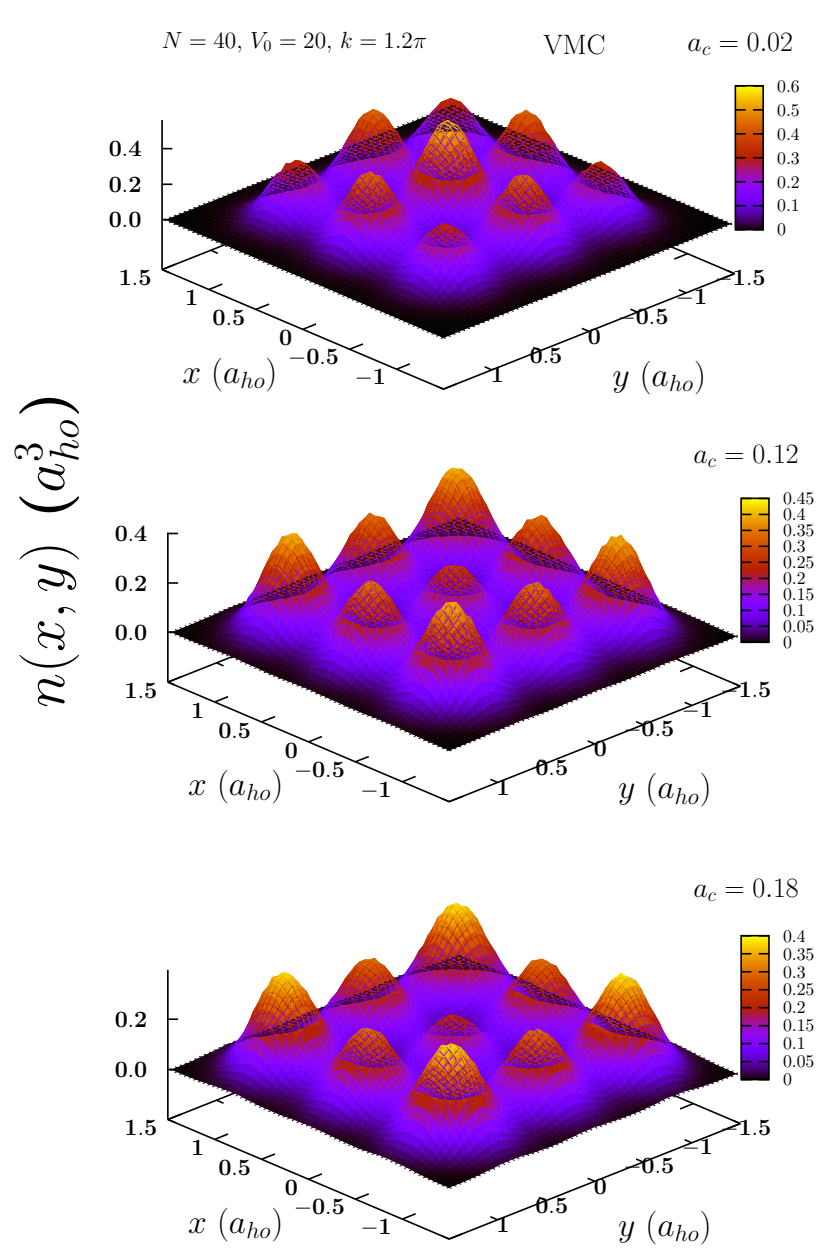

FIG. 20: As in Fig. 16, but for $N=40, V_{0}=20$, and $k=$ $1.2 \pi$.

with an additional parameter $\epsilon$ added to the previous set of variational parameters. Nevertheless, this produces similar results to the previous section. Fig. 22 displays the density slices for the system of $N=8, V_{0}=10$, and $k=\pi$, using Eq. (17). The wave functions in each well do not broaden, but the central peak looses amplitude with increasing $a_{c}$, whereas the peaks at the edges gain amplitude.

\section{SF TO MI PSEUDOTRANSITION}

Although our chief goal was to fix $V_{0}$ and vary $a_{c}$ only, we nevertheless divert a little and explore the evolution of a HS Bose gas in a CHOCL by changing $V_{0}$ and fixing $a_{c}$. The goal is to check whether there is a critical $V_{0}$ at which a SF to MI transition occurs in the presence of an external harmonic trap.

Fig. 23 displays the evolution of the integrated opti-

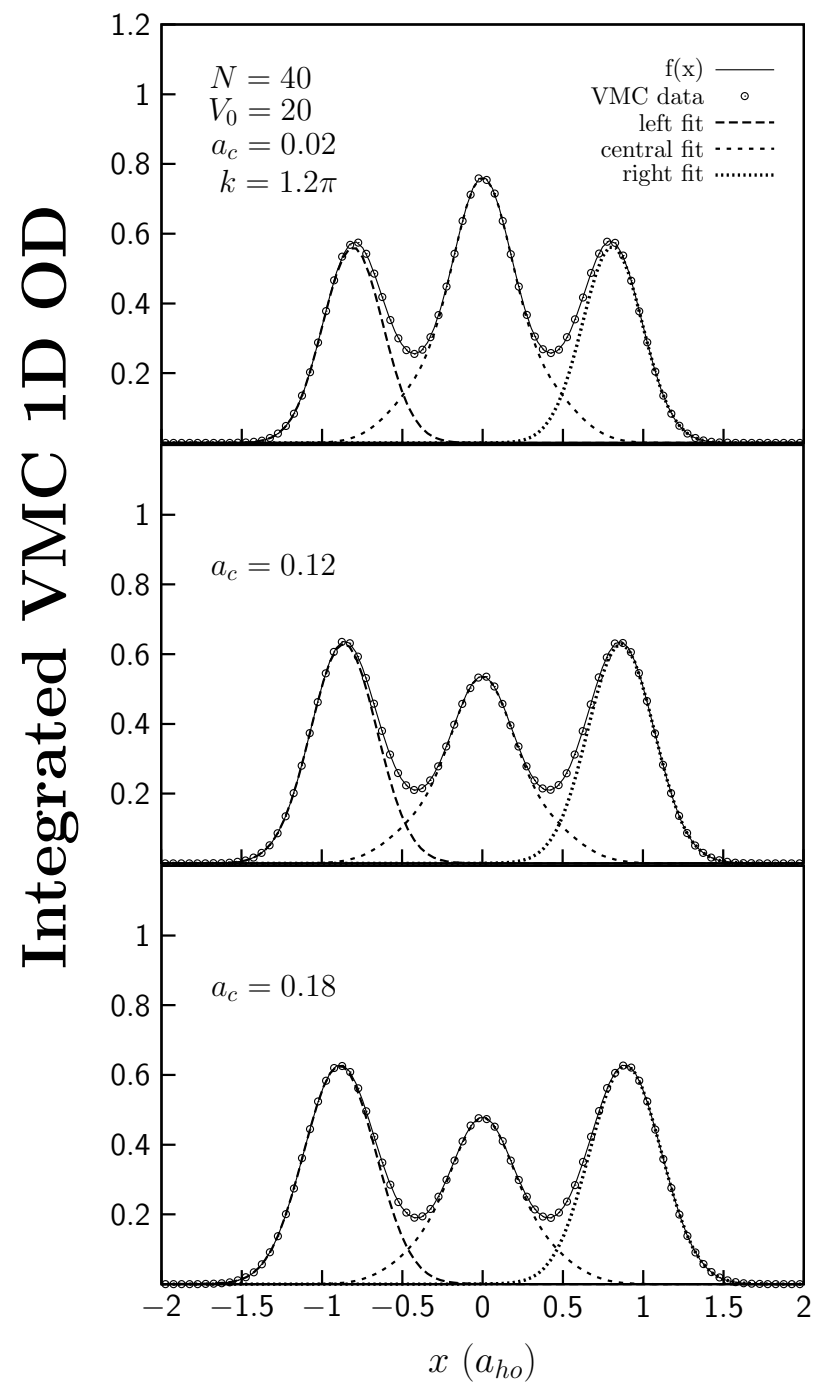

FIG. 21: As in Fig. 17 but for $N=40, V_{0}=20$, and $k=1.2 \pi$

cal density (map view) for a system of $N=8$ particles, $k=\pi$, fixed HS diameter $a_{c}=0.14$, and various optical depths $V_{0}$. From top to bottom: $V_{0}=1,2,6$, and 10, respectively. As $V_{0}$ is increased, the overlap of the wave functions in neighboring wells drops, however, the change is not abrupt but gradual. That is, there does not exist a critical point for a superfluid to Mott-insulator transition. Fig. 24 displays, similarly to Figs. 17 and 19, again the density slices of Fig. 23 along the x-axis, where one can clearly see how the overlap, and therefore the tunneling, drops. We anticipate then, that as $V_{0}$ is increased further, the tunneling will drop substantially. Note, however, that the amplitude of the central peak rises with increasing $V_{0}$. 


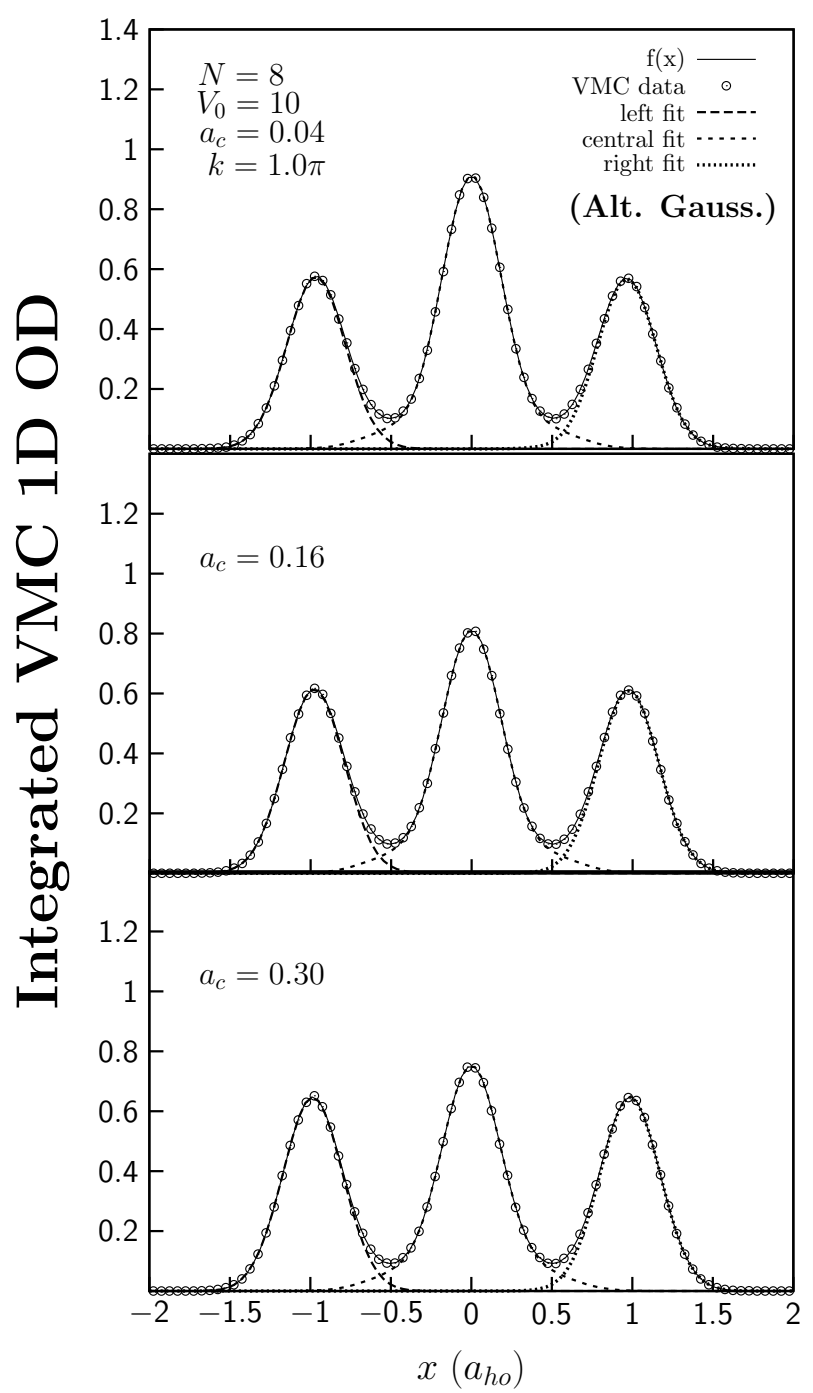

FIG. 22: As in Fig. 19 but using the alternative Gaussian in Eq.17) (Alt. Gauss).

\section{DISCUSSION AND CONCLUSION}

In summary, we have investigated the effects of strongly repulsive interactions on the tunneling amplitude of HS bosons confined in a $3 \mathrm{D}^{a l}$ simple cubic optical lattice plus by a tight spherical harmonic trap. The tunneling amplitude was measured by the overlap integral of the wave functions in neighboring wells. The external harmonic trap was introduced in order to cause an inhomogeneiety in the density distribution of the trapped system. Another goal was also to explore the effect of this inhomogeneiety on the SF to MI transition of bosons in optical lattices.

It was found that for small $\mathrm{N}$, the tunneling amplitude does not change by increasing the boson-boson repulsion at fixed lattice spacing $d=\pi / k$. This is mainly because the width of each localized wave function does not

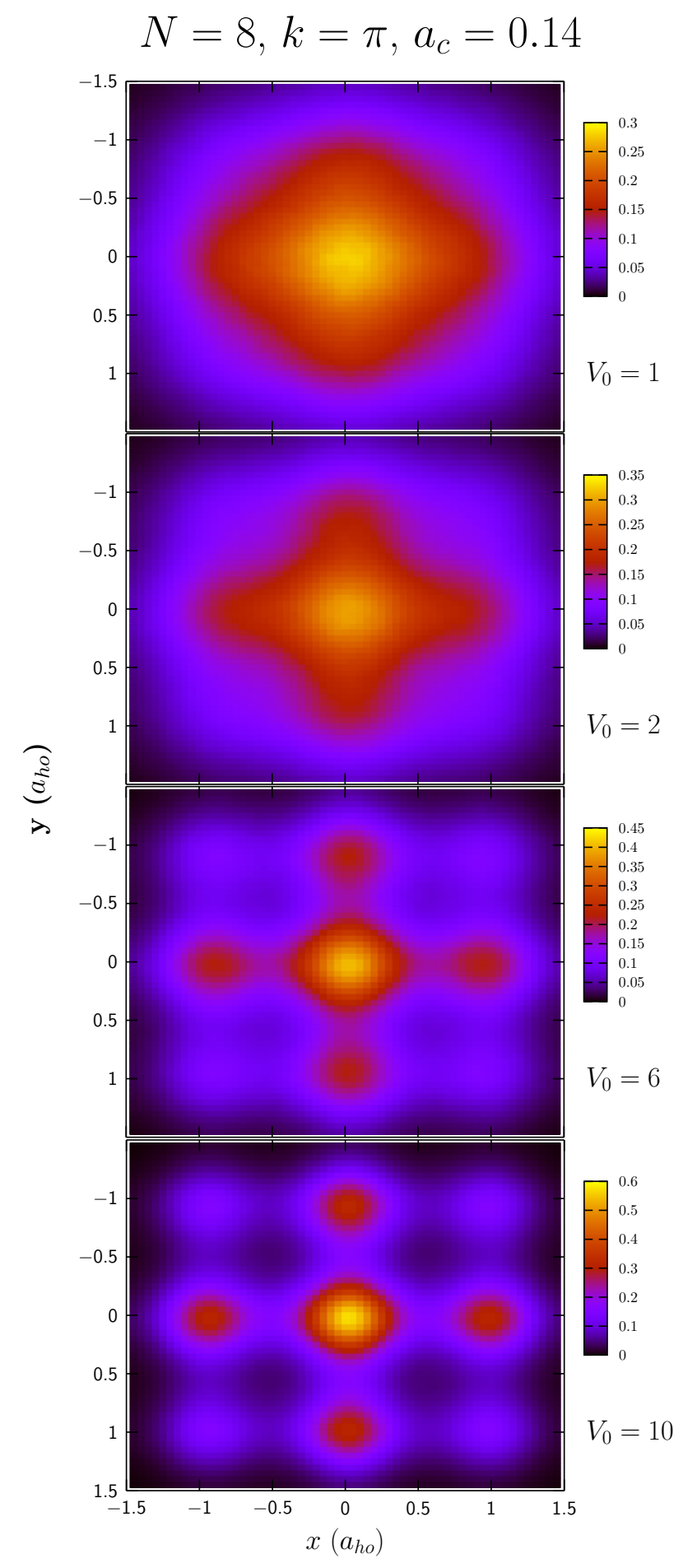

FIG. 23: Map view of integrated VPI optical density for various optical depths $V_{0}$. The system is a HS Bose gas of $N=8$, fixed $a_{c}=0.14$, and $k=1.0 \pi$ which is confined in a CHOCL. From top to bottom: $V_{0}=1,2,6$, and 10. Density and lengths are in trap units.

broaden with a rise in the repulsive forces. The tunneling amplitude, however, rises by reduction of $d$ at fixed $a_{c}$. 


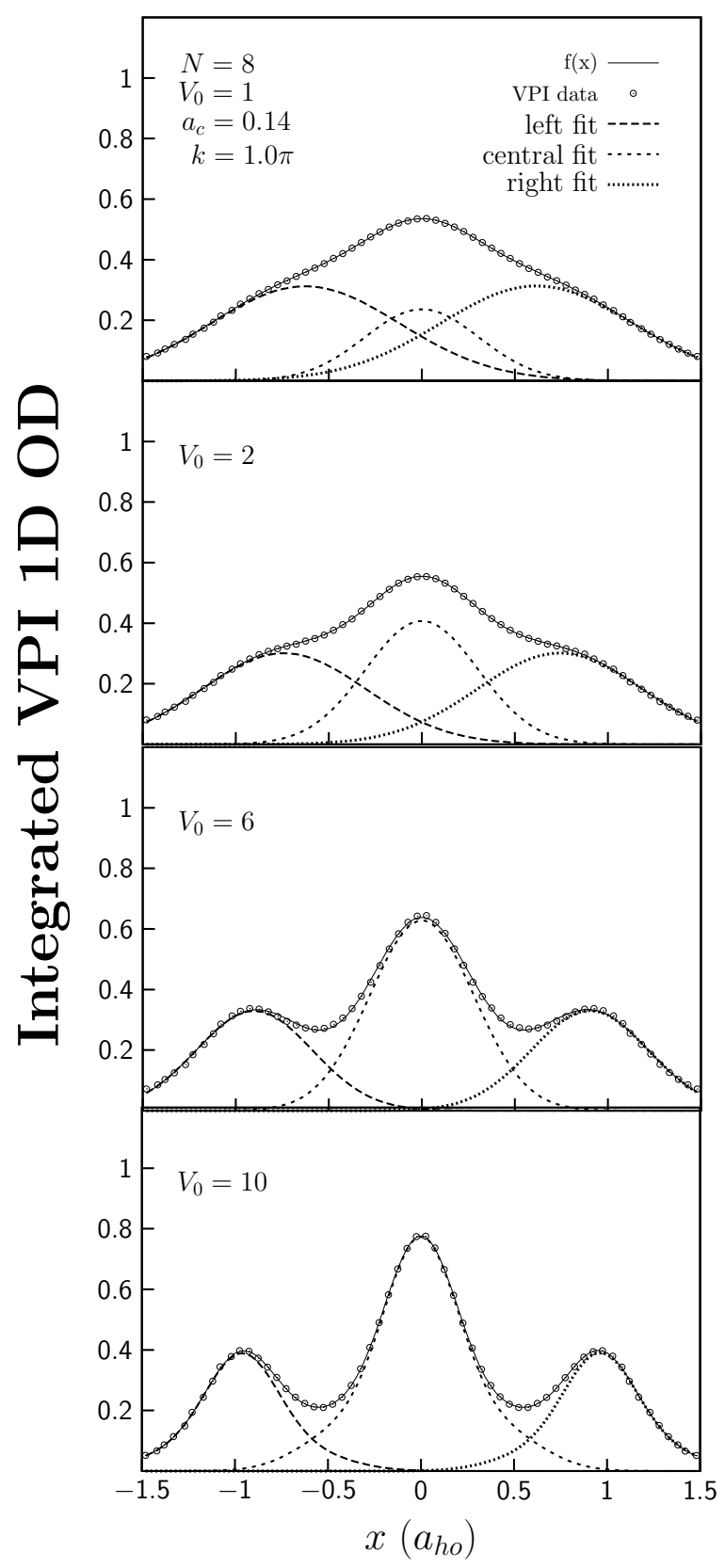

FIG. 24: Density slices of Fig. 23 along the x-axis. The legends describe the same functions as in Fig. 17

Even at very large repulsion, tunneling is still present in the system and does not vanish completely. The presence of an external trap seems to suppress the SF to MI abrupt transition. The transition is gradual and there is no criticality.

The energies change only weakly with increasing $a_{c}$ for $N=2$, but a more pronounced change is observed for $N=8$, and is much larger for $N=40$. The occupancy of the central and first nearest neighbor sites drops with increasing $a_{c}$, and rises at the second and third nearest neighbor sites.

\section{A. Occupancy and onsite interaction energies}

Although the occupancy of particles in Fig. 11 at sites $(000)$ and $(00-1)$ drops with increasing $a_{c}$ causing a drop in the density at the central lattice site, the corresponding $\left\langle U_{(000)}\right\rangle$ and $\left\langle U_{(00-1)}\right\rangle$ still rise. Thus the effect of increasing $g$ via $a_{c}$ on $\left\langle U_{n}\right\rangle$ overwhelms that of the drop in the occupancy at each lattice site. The net result is that $\left\langle U_{(p q r)}\right\rangle$ increases with increasing $g$. Further, the occupancy of the lattice sites plays a crucial role in determining the relative magnitudes of $\left\langle U_{(p q r)}\right\rangle$. Since (000) has the highest occupancy at all $a_{c}$, in Figs. 7 and 8 it has also the highest onsite repulsion. Then with decreasing occupancy, the onsite repulsion decreases in the order $U_{(000)}, U_{(00-1)}, U_{(011)}$, and $U_{(111)}$ at all $a_{c}$. In Fig. 11. one can also see that (000) has the highest, whereas (111) the lowest occupancy.

In equilibrium, one could assume that there are as many particles tunneling into a lattice site as out which preserves the occupancy at each lattice site in equilibrium [49. When $a_{c}$ is changed, the particles relocate themselves in order to reach a new equilibrium configuration. If $a_{c}$ is increased, particles are pushed out of the central site and occupy others until a new equilibrium configuration is reached again. Due to the external trap they are not able to tunnel further away from the edges of the trap. Further, note it is possible that during the equilibration process the particles having tunneled from the center of the trap to the first neighbors continue tunneling to the second and third. In fact, it would be interesting to know how the particles' motion is channelled along the sites of a larger simple cubic optical lattice, as the repulsion between the bosons is increased. Finally, the average (noninteger) occupation numbers $\left\langle N_{(p q r)}\right\rangle$ at each lattice signal that the systems are SF. This indicates also that the particles are fragmented all throughout the lattice.

\section{B. Optical density and tunneling}

As we have seen in Figs. 16- 21, as much as the repulsion was increased, the overlap between neighboring wells did not change significantly, even at large $N$. But the change in the overlap is more pronounced if one increases $V_{0}$ while keeping $a_{c}$ fixed. This was the situation displayed in Fig. 24. We conclude then, that a MI state can be obtained more efficiently by rather increasing the optical depth $V_{0}$ than the HS repulsion between the bosons, $a_{c}$.

Similarly to our findings in Sec III H. Greiner et al. [1] showed, by applying the Bose-Hubbard (BH) model to a $3 \mathrm{D}$ optical lattice, that if the tunneling dominates the BH Hamiltonian, the single particle wave functions are spread over the whole lattice, and phase coherence ex- 
isting between the lattice sites forms a SF. On the other hand, if the atom-atom interactions dominate, there is no phase coherence. Therefore, the single-particle wavefunctions become localized at their lattice sites with a fixed number of atoms, thus forming a MI state. In our case, however, we do not get a pure MI even at very high repulsion because tunneling is still present there. In the highly repulsive regime, we may nevertheless be talking about a quasi MI state where tunneling is present but vanishingly small.

In Fig. 23, if one keeps increasing $V_{0}$, we anticipate that the tunneling amplitude will eventually drop to zero signaling initially what looks like an entrance into the MI regime. However, in order to identify a pure MI regime, integer occupancy of each lattice site is needed. Because the number of particles is less than the number of lattices sites $\left(N / N_{L}<1\right)$, the particles are fragmented [29] and we do not get integer occupancy at each lattice site. This was shown in SecIIIE and as a result this system may be regarded a quasi MI with a small SF component.

\section{The role of the lattice spacing $d=\pi / k$}

In Figs. 2, and 3 the reduction in $\pi / k$ reduces the width of the optical potential barriers, thus reducing the distance a particle needs in order to tunnel completely through a barrier. As a result, an increase in the overlap occurs between two neighboring single-particle wavefunctions $\phi\left(\mathbf{r}, \mathbf{R}_{n}\right)$ and $\phi\left(\mathbf{r}, \mathbf{R}_{n+1}\right)$ causing a rise in $I_{\text {overlap }}$. The increase in $I_{\text {overlap }}$ with $k$ could be further explained on the basis of a recent evaluation of the tunneling rate in 1D through a single, tilted optical barrier without external confinement by Huhtamäki et al. 3. 3. If $a$ and $b$ are the left-hand and right-hand classical turning points of a potential barrier, then if the distance between $a$ and $b$ is reduced by increasing the lattice wave vector $k$, the overlap between the wave functions in neighboring wells rises.

\section{The effects of the external harmonic trap}

The mobility of the bosons is eventually restricted to the confining volume of the external harmonic trap. By increasing the size of the bosons with $a_{c}$, the available free space for motion in the confined volume is reduced. Further, as the repulsion rises with $a_{c}$, the tunneling amplitude remains constant for a small number of particles as they get locked inside the lattice sites. A tight external harmonic potential also suppresses the critical SF to pure MI transition as was shown in Fig. 24. In fact, Gygi et al. [17] argued similarly that the presence of a gradient due to the external confining potential destroys quantum criticality in the transition from the SF to the MI phase; this gradient is present in our simulations. Further, as high as the repulsion between the bosons in our systems becomes, tunneling is still present due to the presence of external confinement. This forces an inhomogeneiety in particle distribution over the lattice sites.

\section{E. Energy}

The rise in $\langle E / N\rangle$ with $k$ in Figs 13 , 14, and 15 , could be attributed to Heisenberg's uncertainty principle. A decline in the available volume the bosons can occupy in the optical lattice wells reduces the uncertainty in their positions. Thus, the uncertainty in their momenta increases and as a result their kinetic energy (quantum pressure) rises. It can also be seen that a change in $k$ has a more profound effect on $E$ than a change in $a_{c}$. Therefore, the lattice dimension seems to play a profound role in determining the SF properties of bosons in optical lattices in that it can substantially control the tunneling amplitude between neighboring wells.

\section{F. Other work}

Similarly to Bach and Rzázewski 24, we used Gaussians of the form $\exp \left[-\alpha\left(x-x_{n}\right)^{2}\right]$ but weighted by a polynomial as in Eq. 67. These authors determined that the mobility of the atoms, as described by the hopping parameter $t$ and their interactions $U$, is controlled by the optical-lattice depth. If this depth is shallow, the atoms become delocalized over the whole lattice as it almost occurs in Fig. 16 .

Li et al. [7] investigated the SF to MI transitions in atomic BECs confined in optical lattices by using the $\mathrm{BH}$ model. Using an isotropic cubic lattice, they have chosen a variational trial function of the form $g(u)=$ $\left(1+\alpha u^{2}\right) \exp \left(-\beta u^{2}\right)$ (a Wannier function); our singleparticle wave function is the same as theirs, except for an additional factor $\propto u^{4}$ [Eq.(6)]. They addressed the possibility of observing SF to MI transitions for an average lattice-site occupancy larger than one. It was noted that by increasing the repulsion between the lattice bosons, their wavefunction in each well broadens, thus enhancing $J$ between neighbouring lattice sites. In contrast, our atomic clouds did not expand due to the presence of external confinement. Li et al. evaluated the on-site interaction energy by variationally minimizing the energy with respect to the parameters $\alpha$ and $\beta$.

Capello et al. 23] used VMC to describe the MI transition (MIT) by means of a variational Gutzwiller wavefunction in discrete space and optimized the variational parameters to achieve the ground state system. In contrast we used a wave function in continuous space and the total energy was minimized in our research as they did.

\section{ACKNOWLEDGEMENT}

Interesting and enlightening discussions with Saverio Moroni and William Mullin are gratefully acknowl- 
edged. We thank William Mullin for a critical reading of the manuscript. One of the authors (ARS), gratefully acknowledges the International Center for Theoret- ical Physics (ICTP) for providing computational access to their GRID cluster facility.
[1] M. Greiner, O. Mandel, T. Esslinger, J. W. Haensch, and I. Bloch, Nature 415, 39 (2002).

[2] K. P. Schmidt, J. Dorier, A. Läuchli, and F. Mila, Phys. Rev. B 74, 174508 (2006).

[3] J. A. M. Huhtamäki, M. Möttönwn, J. Ankerhold, and S. M. M. Virtanen, Phys. Rev. A 76, 033605 (2007).

[4] D.-S. Lühmann, K. Bongs, K. Sengstock, and D. Pfankuche, Phys. Rev. A 77, 023620 (2008).

[5] S. Fölling, S. Trotzky, P. Cheinet, M. Feld, R. Saers, A. Widera, T. Müller, and I. Bloch, Nature 448, 1029 (2007).

[6] Sascha Zöllner, Hans-Dieter Meyer, and Peter Schmelcher, Phys. Rev. Lett. 100, 040401 (2008).

[7] J. Li, Y. Yu, A. M. Dudarev, and Q. Niu, New Journal of Physics 8, 154 (2006).

[8] R. A. Barankov and S. N. Burmistrov, Phys. Rev. A 67, 013611 (2003).

[9] S. R. Clark and D. Jaksch, New Journal of Physics 8, 160 (2006).

[10] P. B. Blackie and W.-X. Wang, Phys. Rev. A 76, 053620 (2007).

[11] G.-D. Lin, W. Zhang, and L.-M. Duan, Phys. Rev. A 77, 043626 (2008).

[12] Ali A. Shams and Henry R. Glyde, Superfluidity and $B E C$ in optical lattices and porous media: a path integral Monte Carlo study (2009).

[13] A. Saito, A. Kawakami, H. Shimakage, H. Terai, and Z. Wang, J. Appl. Phys. 92, 12 (2009).

[14] J. Yin, Phys. Rep. 430, 1 (2006).

[15] J.-Q. Liang, J.-L.Liu, W.-D. Li, and Z.-J. Li, Phys. Rev. A 79, 033617 (2009).

[16] F. Gerbier, A. Widera, S. Fölling, O. Mandel, T. Gericke, and I. Bloch, Phys. Rev. A 72, 053606 (2005).

[17] O. Gygi, H. G. Katzgraber, M. Troyer, S. Wessel, and G. G. Batrouni, Phys. Rev. A 73, 063606 (2006).

[18] K. Mitra, C. J. Williams, and C. A. R. Sa'de Melo, Phys. Rev. A 77, 033607 (2008).

[19] D. Jaksch, C. Bruder, J. I. Cirac, C. W. Gardner, and P. Zoller, Phys. Rev. Lett. 81, 3108 (1998).

[20] E. Timmermans, P. Tommasini, M. Hussein, and A. Kerman, Phys. Rev. 315, 199 (1999).

[21] J. L. DuBois and H. R. Glyde, Phys. Rev. A 63, 023602 (2001).

[22] A. R. Sakhel, J. L. DuBois and H. R. Glyde, Phys. Rev. A 66, 063610 (2002).

[23] M. Capello, F. Becca, M. Fabrizio, and S. Sorella, Phys. Rev. Lett. 99, 056402 (2001).

[24] R. Bach and K. Rzázewski, Phys. Rev. A 70, 063622 (2004).

[25] B. C.-Sansone, E. Kozik, N. Prokof'ev, and B. Svistunov,
Phys. Rev. A 75, 013619 (2007).

[26] M. Yamashita and M. W. Jack, Phys. Rev. A 76, 023606 (2007).

[27] P. B. Blackie, New Journal of Physics 8, 157 (2006).

[28] Y. V. Bludov, J. Santhanam, V. M. Kenkre, and V. V. Konotop, Phys. Rev. A 74, 043620 (2006).

[29] V. V. Konotop and M. Salerno, Phys. Rev. A 65, 021602(R) (2002).

[30] K. Rodriguez, S. R. Manmana, M. Rigol, R. M. Nowak, and A. Muramatsu, New Journal of Physics 8, 169 (2006).

[31] I. Titvinidze, M. Snoek, and W. Hofstetter, Phys. Rev. Lett. 100, 100401 (2008).

[32] G.-P. Zheng, J.-Q. Liang, and W. M. Liu, Phys. Rev. A 71, 053608 (2005).

[33] G. Jin, C. K. Kim, and K. Nahm, Phys. Rev. A 72, 045601 (2005).

[34] H. Heiselberg, Phys. Rev. A 74, 033608 (2006).

[35] B. B. Baizakov, B. A. Malomed, and M. Salerno, Phys. Rev. E 74, 066615 (2006).

[36] R. A. Barankov, C. Lannert, and S. Vishveshwara, Phys. Rev. A 75, 063622 (2007).

[37] M. Rigol and A. Muramatsu, Phys. Rev. A 69, 053612 (2004).

[38] E. Burovski, N. Prokof'ev, B. Svistunov, and M. Troyer, New Journal of Physics 8, 153 (2006).

[39] P. Buonsante, R. Franzosi, and V. Penna, J. Phys. B: At. Mol. Opt. Phys. 37, S195 (2004).

[40] N. V. Prokof'ev, B. V. Svistunov, I. S. Tupitsyn, Phys. Lett. A 238, 253 (1998).

[41] N. Proko'fev, B. Svistunov, and I. Tupitsyn, Phys. Rev. Lett. 82 (1999).

[42] M. Wouters, J. Tempere, and J. T. Devreese, Phys. Rev. A 70, 013616 (2004).

[43] J. E. Cuervo, P.-N. Roy, and M. Boninsegni, J. Chem. Phys. 122, 114504 (2005).

[44] A. Sarsa, K. E. Schmidt, and W. R. Margo, J. Chem. Phys. 113 (2000).

[45] M. H. Kalos and P. A. Whitlock, Monte Carlo Methods, Volume I: Basics (John Wiley and Sons, 1986).

[46] W. H. Press, S. A. Teukolsky, W. T. Vetterling, and B. B. Flannery, Numerical Recipes in $C$ (Cambridge University Press, 1999), 2nd ed.

[47] A. R. Sakhel, J. L. DuBois, and H. R. Glyde, Phys. Rev. A 77, 043627 (2008).

[48] O. Morsch and M. Oberthaler, Rev. Mod. Phys. 78 (2006).

[49] William B. Mullin, University of Massachusetts, Amherst MA, USA. Private communications. 